\title{
Simplicial models of trace spaces
}

\author{
MARTIN RAUSSEN
}

\begin{abstract}
Directed algebraic topology studies topological spaces in which certain directed paths (d-paths) are singled out; in most cases of interest, the reverse path of a d-path is no longer a d-path. We are mainly concerned with spaces of directed paths between given end points, and how those vary under variation of the end points. The original motivation stems from certain models for concurrent computation. So far, homotopy types of spaces of d-paths and their topological invariants have only been determined in cases that were elementary to overlook.

In this paper, we develop a systematic approach describing spaces of directed paths up to homotopy equivalence - as finite prodsimplicial complexes, ie with products of simplices as building blocks. This method makes use of a certain poset category of binary matrices related to a given model space. It applies to a class of directed spaces that arise from a certain class of models of computation - still restricted but with a fair amount of generality. In the final section, we outline a generalization to model spaces known as Higher Dimensional Automata.

In particular, we describe algorithms that allow us to determine not only the fundamental category of such a model space, but all homological invariants of spaces of directed paths within it. The prodsimplical complexes and their associated chain complexes are finite, but they will, in general, have a huge number of cells and generators.
\end{abstract}

55P10, 55P15, 55U10; 68Q55, 68Q85

\section{Introduction}

\subsection{Background}

With motivations arising originally from concurrency theory within computer science, a new field of research, directed algebraic topology, has emerged; for a comprehensive overview from a categorical perspective, we refer to the recent book by Grandis [13]. Directed algebraic topology involves spaces of "directed paths" (or timed paths, executions): these directed paths can be concatenated, but in general not reversed; time is not reversible. 
A particular model for concurrent computation, called Higher Dimensional Automata (HDA), was introduced by V Pratt [23] back in 1991. Mathematically, HDA can be described as (labelled) precubical sets (cf Brown and Higgins $[2 ; 1]$ ) with a preferred set of directed paths respecting the natural partial orders in any of the cubes of the model; (di-)homotopies of such directed paths have to respect the order along a deformation; cf Fajstrup, Goubault and Raussen [6].

Compared to other well-studied concurrency models like labelled transition systems, event structures, Petri nets etc (for a survey on those, see Winskel and Nielsen [30]), it has been shown by R J van Glabbeek [9] that Higher Dimensional Automata have the highest expressivity; on the other hand, they are certainly less studied and less often applied so far.

All concurrency models deal with sets of states and with sets of execution paths - with some further structure. The interest is mainly in the path spaces; typically, it is difficult to get an overview and to infer valuable information about the path space from the state space model.

A general framework for topological spaces with directed paths was defined and investigated as the category of $\mathrm{d}$-spaces ( $\mathrm{d}$ for directed). The objects are topological spaces with a preferred set of d-paths; the morphisms are the continuous maps preserving d-paths; cf in particular Grandis $[12 ; 11 ; 13]$. Grandis investigates first of all the fundamental category of a d-space - generalising the fundamental group of a topological space. Unlike the classical case, spaces of d-paths depend critically on the chosen end points. This makes it interesting to investigate how spaces of d-paths (with given fixed end points) vary under variation of these end points (cf Raussen [26]) and how this gives rise to a suitable decomposition of the state space into "components"; cf Fajstrup, Goubault, Haucourt and Raussen [7; 10].

General topological properties of spaces of d-paths and of traces (=d-paths up to monotone reparametrizations; cf Fahrenberg and Raussen [4; 27]) in semicubical complexes were investigated in Raussen [28]. But so far, apart from low-dimensional examples with convincing drawings, there have been very few explicit examples of actual computations of spaces of such traces (for an attempt in dimension two, see Raussen [24]); let alone a general method to perform such computations.

It is the aim of this article to make the homotopy types of trace spaces computable for a restricted class of Higher Dimensional Automata - those arising from the semaphore or PV-models introduced by Dijkstra [3] back in 1968. The state spaces for such models are complements of a number of hyperrectangular "holes" in a partially ordered hypercube $\vec{I}^{n}$. We describe trace spaces for these models explicitly as finite-dimensional 
prodsimplicial complexes (cf Kozlov [20]; with products of simplices as their building blocks) with the nerve of a particular poset category as barycentric subdivision.

For applications in concurrency, it is already very important to know the Betti number $\beta_{0}$ and to get hold on the connected components of a trace space: The reason is that traces in each connected component will always lead to the same result in a concurrent computation. Using the prodsimplicial structure makes it possible - at least in principle, the complexes may have lots of cells - to calculate algebraic topological invariants of such trace spaces.

We will finally hint on how to extend our results to general HDA. The overall philosophy reminds of the analysis of the topology of path spaces in CW-complexes in Milnor's article [22]: Also the spaces of d-paths in a precubical complex with given end points are equi locally convex (ELCX) (cf Raussen [28]) and thus locally contractible; for the PV-models analysed here, suitably chosen contractible subsets can be described explicitly, by a blend of order and combinatorics. They and their intersections form a poset category with a geometric realization that is homotopy equivalent to the path space under consideration.

Note that Jardine [16], using a different approach, has recently described a method to calculate the path category $P(X)$ of a simplicial or cubical set $X$ as the path component category of a related explicitly constructed 2-category. It is a challenge to compare the two methods.

\subsection{Structure and overview of results}

Dijkstra's PV-models [3] are a particular class of models for linear concurrent computations with semaphores, a particularly simple, but instructive class of Higher Dimensional Automata. These models are introduced in Section 2; the state space $X$ for such a model is embedded in a hypercube $\vec{I}^{n}$ and inherits a partial order. To get going, we define certain subspaces of the model space and show that the space of d-paths within any of these subspaces (for simplicity, from the bottom $\mathbf{0} \in \vec{I}^{n}$ to the top $\mathbf{1} \in \vec{I}^{n}$ ) is empty or contractible by a specific contraction. Moreover we show, that every d-path in the model space is contained in at least one of these subspaces.

This allows us in Section 3 to define a poset category $\mathcal{C}(X)(\mathbf{0}, \mathbf{1})$ indexing the nonempty subspaces of restricted d-paths in $X$ (for simplicity, with paths starting at $\mathbf{0}$ and ending at 1) described above and their nonempty intersections. That category is naturally isomorphic to a poset subcategory of a product of a number of order categories of nonempty subsets of the positive integers $[1: n]$ less than or equal to $n$. A topological realization of this subcategory can thus be modelled on products of 
simplices and gives rise to a prodsimplicial complex [20]. Using standard methods (nerve lemma, projection lemma etc; cf Kozlov [20]), we show that the space of d-paths (or rather traces, ie, d-paths modulo monotone reparametrizations; cf Fahrenberg and Raussen [4]) $\vec{T}(X)(\mathbf{0}, \mathbf{1})$ in such a model space is in fact homotopy equivalent to an explicit prodsimplicial complex $\mathbf{T}(X)(\mathbf{0}, \mathbf{1})$ that arises as geometric realization of the poset category $\mathcal{C}(X)(\mathbf{0}, \mathbf{1})$ - with the nerve $\Delta(\mathcal{C}(X)(\mathbf{0}, \mathbf{1}))$ of that category as barycentric subdivision.

It is the aim of Section 4 to exploit this theoretical result by achieving an explicit description of the index category $\mathcal{C}(X)(\mathbf{0}, \mathbf{1})$. To this end, it is necessary to decide, for each of the subspaces mentioned above, whether it is empty or not, ie whether there exists a d-path within it from bottom to top. Every subspace can be described as the complement of a number $l$ of homothetic hyperrectangles (with faces parallel to the coordinate planes) extending the original holes. It turns out that it is enough to find out whether there exist deadlock points (the only d-path with a deadlock as source is trivial) in these extended models. A combinatorial search algorithm for deadlocks was earlier described in Fajstrup, Goubault and Raussen [5]. The outcome of a systematic search for deadlocks (in all extended models) is a set $D(X)(\mathbf{0 , 1})$ of minimal nonfaces - all of dimension $n-1-$ of the prodsimplicial complex $\mathbf{T}(X)(\mathbf{0}, \mathbf{1})$ within the prodsimplicial complex $\left(\Delta^{n-1}\right)^{l}$. The maximal faces of $\mathbf{T}(X)(\mathbf{0}, \mathbf{1})$ can then be determined via minimal transversals in an associated hypergraph.

The explicit determination of the complex $\mathbf{T}(X)(\mathbf{0}, \mathbf{1})$ thus achieved makes the calculation of algebraic topological invariants of the trace space $\vec{T}(X)(\mathbf{0}, \mathbf{1})$ possible. Even if, for complicated model spaces, the "curse of dimensionality" might prohibit explicit calculations, it will still be interesting and possible to study the change of invariants under change of end points (in rounds of computation; compare Herlihy and Rajsbaum [15] and other sources in distributed computing for this point of view).

For simplicity of the presentation, we restrict in this paper attention to an investigation of the traces from the bottom corner $\mathbf{0}$ to the top corner $\mathbf{1}$ in a state space without holes on the boundary $\partial I^{n} \subset I^{n}$. More general situations are important: First of all, most semaphore models allow holes intersecting the boundary $\partial I^{n}$. A study of the fundamental category as in Grandis [12] or of associated categories modelling higher invariants as in Raussen [26] of the model space relies on information about the topology of general spaces $\vec{T}(X)(\mathbf{c}, \mathbf{d})$ at intermediate points $\mathbf{c}, \mathbf{d} \in \vec{I}^{n}$. A modification of the setup discussed here has been described in Raussen [29]; it will be published elsewhere.

The final Section 5 takes first steps in generalizing the methods described so far. Dijkstra's PV-models can easily be generalized to a state space that is a product of 
digraphs with "hyperrectangular holes" modelling processes that may branch, merge and loop. For these, the topology of the trace space can be determined in two steps: First determine (the components of) the traces in the product of digraphs without holes. That space is homotopy equivalent to a product of trace spaces of the 1-dimensional digraphs; it is thus homotopy discrete. For each of the components, one can pull back (or "unloop") to a state space, including holes, of the type previously investigated. It will still have to be investigated how to unloop in a coherent manner in order to reuse calculations (of deadlocks etc) performed during previous steps.

For general HDA (modelled on general precubical sets), it is no longer possible to use the explicit contraction method for specific subspaces yielding local contractability used in this article. Instead, it is probably necessary to use the method described in Raussen [28] with a higher combinatorial complexity still to be sorted out.

\section{Models of computation and subspaces}

\subsection{A simple higher dimensional automaton}

To start with, we analyse spaces of directed paths in a simple model space that can be described as follows: A (linear) schedule for each of a number of $n$ individual processors $P_{j}, 1 \leq j \leq n$, is modelled on the directed interval $\vec{I}_{j}=[0,1]$. On subintervals $\left.I_{j}^{i}=\right] a_{j}^{i}, b_{j}^{i}\left[\subseteq I_{j}, 1 \leq i \leq l\right.$, there is potential conflict with the schedules of the other processors. Let $\mathbf{a}^{i}=\left(a_{1}^{i}, \ldots, a_{n}^{i}\right), \mathbf{b}^{i}=\left(b_{1}^{i}, \ldots, b_{n}^{i}\right) \in I^{n} \backslash \partial I^{n}$ and let $R^{i}=\left\{\mathbf{x} \in I^{n} \mid a_{j}^{i}<x_{j}<b_{j}^{i}, 1 \leq j \leq n\right\}$ denote the "homothetic" hyperrectangle (faces parallel to the coordinate planes) with bottom corner a and top corner $\mathbf{b}$.

The state space for concurrent executions of $n$ linear processes is then the space $X=\vec{I}^{n} \backslash F \subset \vec{I}^{n}$ excluding the forbidden region $F=\bigcup_{i=1}^{l} R^{i}$. The forbidden region $F$ models conflicts and may not be entered. Consult Figure 1 for a simple 3-dimensional example.

The space $X$ inherits a partial order $\leq$ from the componentwise partial order $\leq$ on $\vec{I}^{n}$. We study compound schedules (execution paths) in such a state space $X$ : Ad-path in $X$ is a continuous path $p: \vec{I} \rightarrow X$ that is continuous and order-preserving: each coordinate $\pi_{j} \circ p: \vec{I} \rightarrow X \subset \vec{I}^{n} \rightarrow \vec{I}, 1 \leq j \leq n$, is weakly increasing. The set $\vec{P}(X)(\mathbf{c}, \mathbf{d})$ consists of all d-paths in $X$ starting at $\mathbf{c} \in X$ and ending at $\mathbf{d} \in X$; in particular, these d-paths avoid the "forbidden region" $F \subset \vec{I}^{n}$. Consult eg Gunawardena [14] and Fajstrup, Goubault and Raussen [5] for detailed descriptions.

As a topological space, $\vec{P}(X)(\mathbf{c}, \mathbf{d})$ is given the subspace topology inherited from the space $P(X)(\mathbf{c}, \mathbf{d})=[(I, 0,1) ;(X, \mathbf{c}, \mathbf{d})]$ of all paths in $X$ from $\mathbf{c}$ to $\mathbf{d}$ in the compact-open topology (= uniform convergence topology). 


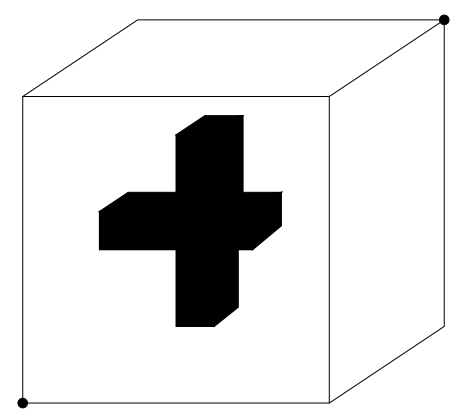

Figure 1: Three-dimensional state space $X$ as complement of a forbidden region $F$ consisting of two hyperrectangles within a unit cube

Reparametrization equivalent d-paths [4] in $X$ have the same directed image (= trace) in $X$. Dividing out the action of the monoid of (weakly-increasing) reparametrizations of the parameter interval $\vec{I}$, we arrive at trace space $\vec{T}(X)(\mathbf{c}, \mathbf{d})$ (cf Fahrenberg and Raussen [4; 27]) which is shown in Raussen [28] to be homotopy equivalent to path space $\vec{P}(X)(\mathbf{c}, \mathbf{d})$ for a far wider class of directed spaces $X$. In the latter paper, it is also shown that trace spaces enjoy nice properties: They are metrizable, locally compact, locally contractible, and they have the homotopy type of a CW-complex.

It is the aim of the present paper to describe and analyze a combinatorial/topological model of spaces of traces (and hence also of d-paths) in a model space $X$ up to homotopy equivalence in order to make calculations of their algebraic topological invariants feasible.

\subsection{Subspaces of the model space}

We will now describe certain subspaces of $X$ and then prove that associated spaces of d-paths within these subspaces are either empty or contractible.

We use the following notation:

- The set of elements "below" $\mathbf{d}=\left(d_{1}, \ldots d_{n}\right) \in X$ is denoted

$$
\downarrow \mathbf{d}:=\{\mathbf{x} \in X \mid \mathbf{x} \leq \mathbf{d}\}=\left\{\mathbf{x} \in I^{n} \mid \mathbf{x} \leq \mathbf{d}, \mathbf{x} \notin F\right\} .
$$

Remark that it is not always possible to reach $\mathbf{d}$ from every $\mathbf{x} \in \downarrow \mathbf{d}$ by a d-path. Likewise $\uparrow \mathbf{c}=\{\mathbf{x} \in X \mid \mathbf{c} \leq \mathbf{x}\}$ denotes the set of elements "above" $\mathbf{c}$.

- The upper boundary $\left\{\mathbf{x} \in \downarrow \mathbf{d} \mid \exists 1 \leq i \leq n: x_{i}=d_{i}\right\}$ of the hyperrectangle with upmost vertex in $\mathbf{d}$ with $X$ will be denoted $\partial_{+} \downarrow \mathbf{d}$.

- $\mathbf{a}^{i}=\left(a_{1}^{i}, \ldots, a_{n}^{i}\right), \mathbf{b}^{i}=\left(b_{1}^{i}, \ldots, b_{n}^{i}\right)$. 
Consider Example 2.3 and Figure 2 for 2-dimensional illustrations of the following definition:

Definition 2.1 (1) For $1 \leq i \leq l, 1 \leq j_{i} \leq n$, let

$$
X_{j_{1}, \ldots, j_{l}}:=\left\{\mathbf{x} \in X \mid \forall i: x_{j_{i}} \leq a_{j_{i}}^{i} \text { or } \exists k: x_{k} \geq b_{k}^{i}\right\} .
$$

(2) For nonempty subsets $J_{i} \subseteq[1: n], 1 \leq i \leq l$, let

$$
X_{J_{1}, \ldots, J_{l}}:=\left\{\mathbf{x} \in X \mid \forall i: x_{j_{i}}^{i} \leq a_{j_{i}}^{i}, j_{i} \in J_{i} \text {, or } \exists k: x_{k} \geq b_{k}^{i}\right\} .
$$

For later use, we note an equivalent formulation of these conditions:

$$
\begin{aligned}
& \mathbf{x} \in X, \forall i:\left(\forall k x_{k}<b_{k}^{i} \Rightarrow x_{j_{i}} \leq a_{j_{i}}^{i}\right) \\
& \mathbf{x} \in X, \forall i:\left(\forall k x_{k}<b_{k}^{i} \Rightarrow x_{j_{i}} \leq a_{j_{i}}^{i}\left(\text { for all } j \in I_{j}\right)\right) .
\end{aligned}
$$

Remark 2.2 An execution path in $X_{j_{1}, \ldots, j_{l}}$ has the following characterization: Processor $j_{i}$ is late at $R^{i}$ : it has not yet reached the "conflict" interval $J_{j_{i}}^{i}$ when one of the others, say $k_{i}$, has already left the corresponding conflict interval $J_{k_{i}}^{i}$.

Example 2.3 Figure 2 shows in each of the two rows an example of a model space $X=\vec{I}^{2} \backslash F$ given as the complement of the forbidden region $F$ consisting of two black squares. The grey-shaded areas show, in both cases, the subspaces $X_{11}, X_{12}, X_{21}$, resp. $X_{22}$, in that order. Remark that an empty space of d-paths $\vec{P}\left(X_{i j}\right)(\mathbf{0}, \mathbf{1})=\varnothing$ occurs only in the second row - and only for $X_{12}$.
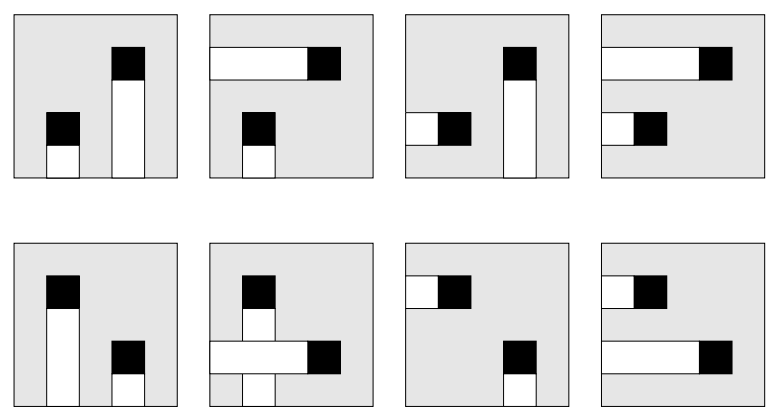

Figure 2: Two examples of a model space $X$ and of subspaces $X_{i j}, 1 \leq$ $i, j \leq 2$, the grey-shaded areas

Example 2.4 In Figure 3, $X=\vec{I}^{3} \backslash F, F=\vec{J}^{3}$ and $\vec{J} \subset \vec{I}$ is an interior open interval. Apart from the forbidden region "black box" $\vec{J}^{3}$ with upper corner $\mathbf{b}$, you see the shaded areas $X_{j} \cap \partial_{+} \downarrow \mathbf{b}, 1 \leq j \leq 3$. Remark that every pair $X_{j_{1}}, X_{j_{2}}$ of these areas intersect, whereas the intersection $X_{1} \cap X_{2} \cap X_{3}$ is empty. In particular, $\vec{P}\left(X_{J}\right)(\mathbf{0}, \mathbf{1})=\varnothing$ for $\varnothing \neq J \subseteq[1: 3]$ if and only if $J=[1: 3]$. 

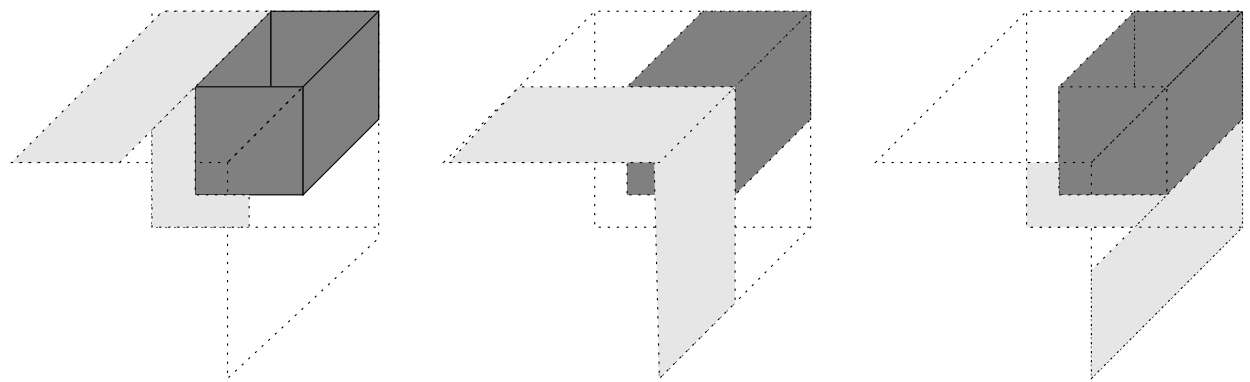

Figure 3: Intersections of $X_{i}$ with the upper boundary $\partial_{+} \downarrow \mathbf{b}$ of the box with upper corner $\mathbf{b}$

The subspaces from Definition 2.1 above have the following obvious property:

Lemma 2.5 $X_{J_{1}, \ldots, J_{l}}=\bigcap_{j_{i} \in J_{i}} X_{j_{1}, \ldots, j_{l}}$.

\subsection{Restricted path spaces are empty or contractible}

With $\mathbf{a}=\left(a_{1}, \ldots, a_{n}\right), \mathbf{b}=\left(b_{1}, \ldots, b_{n}\right)$, the binary operation $\vee$ on $\mathbf{R}^{n}$ (the least upper bound) is given by $\mathbf{a} \vee \mathbf{b}=\left(\max \left(a_{1}, b_{1}\right), \ldots, \max \left(a_{n}, b_{n}\right)\right)$. Observe ((1) as a consequence of Definition 2.1):

Lemma 2.6 (1) $X_{j_{1}, \ldots, j_{l}}$ is closed under $\vee$ for every choice $j_{i} \in[1: n], i \in[1: l]$.

(2) Intersections of $\vee$-closed sets are $\vee$-closed.

(3) $X_{J_{1}, \ldots, J_{l}}$ is closed under $\vee$ for every collection of nonempty subsets $J_{i} \subseteq[1: n]$, $1 \leq i \leq l$.

A similar result holds for the binary operation $\wedge$ (the greatest lower bound) given by $\mathbf{a} \wedge \mathbf{b}=\left(\min \left(a_{1}, b_{1}\right), \ldots, \min \left(a_{n}, b_{n}\right)\right)$ after a suitable change of definition for $X_{j_{1}, \ldots, j_{l}}$.

Note that Lemma 2.6 no longer holds if one of the sets $J_{i}$ may be empty!

Remark 2.7 It should also be possible to exploit the least upper bound operation $\vee$ for the definition and analysis of (future) components (cf Fajstrup, Goubault, Haucourt and Raussen [7; 10]) as follows: $\mathbf{x}, \mathbf{y} \in X$ are elementarily future related if $\mathbf{z}_{1} \vee \mathbf{z}_{2} \in X$, for every $\mathbf{z}_{1}, \mathbf{z}_{2} \in X$ with $\vec{P}(X)\left(\mathbf{x}, \mathbf{z}_{i}\right) \neq \varnothing \neq \vec{P}(X)\left(\mathbf{z}_{i}, \mathbf{y}\right)$. Consider the equivalence relation future equivalent generated by symmetric and transitive closure. This idea will be pursued elsewhere.

The next observation that is valid for more general binary operations $*$ is essential for our purposes: 
Proposition 2.8 Assume $A \subseteq X, \mathbf{a}, \mathbf{b} \in A$.

Let $*: A \times A \rightarrow A$ denote a commutative continuous map satisfying

- $\mathbf{x}_{i} \leq \mathbf{y}_{i} \Rightarrow \mathbf{x}_{1} * \mathbf{x}_{2} \leq \mathbf{y}_{1} * \mathbf{y}_{2}$, ie, $*$ is a d-map;

- $\mathbf{x} \leq \mathbf{y} \in A \Rightarrow \mathbf{x} * \mathbf{y}=\mathbf{y}$.

Then the following holds:

(1) The trace space $\vec{T}(A)(\mathbf{c}, \mathbf{d})$ is either empty or contractible.

(2) Let $\varnothing \neq J_{i} \subseteq[1: n], 1 \leq i \leq l$ and $\mathbf{c}, \mathbf{d} \in X_{J_{1}, \ldots J_{l}}$. Then the trace space $\vec{T}\left(X_{J_{1}, \ldots J_{l}}\right)(\mathbf{c}, \mathbf{d})$ is either empty or contractible.

Proof (2) follows from (1) and Lemma 2.6 with $*=\vee$.

To prove (1), we show first that $\vec{P}(A)(\mathbf{c}, \mathbf{d})$ is either contractible or empty. If $\vec{P}(A)(\mathbf{c}, \mathbf{d})$ is nonempty, then, for any pair $p, q \in \vec{P}(A)(\mathbf{c}, \mathbf{d})$, define a one-parameter family $H(p, q): \vec{P}(A)(\mathbf{c}, \mathbf{d}) \times I \rightarrow \vec{P}(A)(\mathbf{c}, \mathbf{d})$ by

$$
H_{t}(p, q)(s):=q(s) * p(t s), t \in I .
$$

Remark that $H_{0}(p, q)(s)=q(s) * \mathbf{c}=q(s), H_{t}(p, q)(0)=\mathbf{c} * \mathbf{c}=\mathbf{c}, H_{t}(p, q)(1)=$ $\mathbf{d} * p(t)=\mathbf{d}$ and that $H_{1}(p, q)(s)=q(s) * p(s)$. Thus $H(p, q)$ defines an increasing d-homotopy (cf Grandis [12]) $q \mapsto p * q$ between d-paths in $\vec{P}(A)(\mathbf{c}, \mathbf{d})$. Likewise, $H(q, p)$ is an increasing d-homotopy $p \mapsto q * p=p * q$. Their concatenation $G(q, p)=$ $H(p, q) * H^{-}(q, p)$ (orientations are reversed for the second d-homotopy) is a "zig-zag" d-homotopy from $q$ to $p$; in particular a path from $q$ to $p$ within $\vec{P}(\mathbf{c}, \mathbf{d})$. The map $G(-,-)$ defines a continuous section of the "end path map" $\mathrm{ev}_{0} \times \mathrm{ev}_{1}: \vec{P}(\mathbf{c}, \mathbf{d}){ }^{I} \rightarrow$ $\vec{P}(\mathbf{c}, \mathbf{d}) \times \vec{P}(\mathbf{c}, \mathbf{d})$ that associates to a pair $(q, p)$ the d-homotopy $G(p, q)$.

Given an arbitrary $p \in \vec{P}(A)(\mathbf{c}, \mathbf{d})$, the map $G(-, p): \vec{P}(\mathbf{c}, \mathbf{d}) \times I \rightarrow \vec{P}(\mathbf{c}, \mathbf{d})$ is a contraction of $\vec{P}(\mathbf{c}, \mathbf{d})$ to $p$. By Raussen [28], Proposition 2.16, the trace space $\vec{T}(\mathbf{c}, \mathbf{d})$ is homotopy equivalent to the space of d-paths $\vec{P}(\mathbf{c}, \mathbf{d})$ and is thus also contractible.

Remark 2.9 (1) A similar result holds for a map $*$ satisfying $\mathbf{x} \leq \mathbf{y} \Rightarrow \mathbf{x} * \mathbf{y}=\mathbf{x}$.

(2) If $J_{i}=[1: n]$ for at least one $i$, then $\vec{T}\left(X_{J_{1}, \ldots, J_{l}}\right)(\mathbf{0}, \mathbf{1})$ is always empty; in this case, condition (2) from Definition 2.1 amounts to $\mathbf{x}<\mathbf{b}^{i} \Rightarrow \mathbf{x} \leq \mathbf{a}^{i}$. But every d-path from $\mathbf{0}$ to $\mathbf{1}$ needs to pass through the region $\downarrow \mathbf{b}^{i} \backslash\left(\downarrow \mathbf{a}^{i}\right)$ in between.

The trace spaces considered above cover the total trace space: With notation as in Proposition 2.8, we obtain:

Lemma 2.10 $\vec{T}(X)(\mathbf{c}, \mathbf{d})=\bigcup_{[1: n]^{l}} \vec{T}\left(X_{j_{1}, \ldots, j_{l}}\right)(\mathbf{c}, \mathbf{d})$ for any $\mathbf{c}, \mathbf{d} \in X$. 
Proof For a given d-path $p=\left(p_{1}, \ldots, p_{n}\right) \in \vec{P}(X)(\mathbf{c}, \mathbf{d}) \subseteq \vec{P}\left(\vec{I}^{n}\right)(\mathbf{c}, \mathbf{d})$ and $1 \leq i \leq l$, choose a minimal $t_{i}$ such that there exists $k_{i} \in[1: n]$ with $p_{k_{i}}\left(t_{i}\right)=\widetilde{b}_{k_{i}}^{i}:=\min \left(b_{k_{i}}^{i}, \bar{d}_{i}\right)$. If $\tilde{b}_{k_{i}}^{i} \leq a_{k_{i}}^{i}$, then $j_{i} \in[1: n]$ can be chosen arbitrarily; otherwise choose $s_{i}<t_{i}$ such that $p_{k_{i}}\left(s_{i}, t_{i}[)=\right] a_{k_{i}}^{i}, \tilde{b}_{k_{i}}^{i}$. Since $p(t) \notin R^{i}$ for every $t$ and $p_{j}(t)<b_{j}^{i}$ for all $(j, t)$ with $t<t_{i}$, there exists $j_{i}$ such that $p_{j_{i}}(t) \leq a_{j_{i}}^{i}$ for $s_{i}<t<t_{i}$ and hence, by monotonicity, for $t<t_{i}$. In conclusion, $p \in \vec{P}\left(X_{j_{1}, \ldots, j_{l}}\right)(\mathbf{c}, \mathbf{d})$.

In the following Section 3, we need to cover a trace space $\vec{T}(X)(\mathbf{c}, \mathbf{d})$ by open subsets. Therefore, we carefully augment the spaces $X_{J_{1}, \ldots, J_{l}}$ : Choose $\varepsilon>0$ such that all distances $\left|a_{j}^{i}-a_{j}^{k}\right|,\left|a_{j}^{i}-b_{j}^{k}\right|,\left|b_{j}^{i}-a_{j}^{k}\right|>4 \varepsilon$ unless they vanish.

Definition 2.11 $X_{j_{1}, \ldots, j_{l}}$.

(2) $Y_{J_{1}, \ldots, J_{l}}=\bigcap_{j_{i} \in J_{i}} Y_{j_{1}, \ldots, j_{l}} \supset X_{J_{1}, \ldots, J_{l}}$.

Proposition 2.12 Suppose that, for every $1 \leq j \leq n$, no upper boundary $b_{j}^{i}$ is equal to a lower boundary $a_{j}^{k}$; ie, that $\left\{a_{j}^{i}\right\}_{i} \cap\left\{b_{j}^{i}\right\}_{i}=\varnothing$ for every $j$.

(1) There exists a d-map $\varphi: X \rightarrow X$ (continuous and order preserving) and a $d$ homotopy (cf Grandis [12]) $\Phi=\left(\Phi_{t}\right): X \times \vec{I} \rightarrow X, \varphi \rightarrow \mathrm{id}_{X}$ keeping $X_{j_{1}, \ldots, j_{l}}$ pointwise fix that satisfy

$$
\varphi\left(Y_{J_{1}, \ldots, J_{l}}\right) \subseteq X_{J_{1}, \ldots, J_{l}} \quad \text { and } \quad \Phi\left(Y_{J_{1}, \ldots, J_{l}} \times I\right) \subseteq Y_{J_{1}, \ldots, J_{l}}
$$

for all $\left(J_{1}, \ldots, J_{l}\right) \subseteq[1: n]^{l}$.

(2) $X_{j_{1}, \ldots, j_{l}}$ is a deformation retract of $Y_{j_{1}, \ldots, j_{l}}$.

(3) $X_{J_{1}, \ldots, J_{l}}$ is a deformation retract of $Y_{J_{1}, \ldots, J_{l}}$.

Proof Choose weakly increasing reparametrizations $\varphi_{j}: \vec{I} \rightarrow \vec{I}, 1 \leq j \leq n$, of the unit interval $I$ that are piecewise linear, equal to the identity map outside the intervals ]$a_{j}^{i}, a_{j}^{i}+2 \varepsilon$ [ and $] b_{j}^{i}-2 \varepsilon, b_{j}\left[\right.$ and that map the intervals $\left[a_{j}^{i}, a_{j_{\rightarrow}}^{i}+\varepsilon\right]$ constantly to $a_{j}^{i}$ and $\left[b_{j}^{i}-\varepsilon, b_{j}^{i}\right]$ constantly to $b_{j}^{i}$. The product $\varphi=\prod_{j=1}^{n} \varphi_{j}: \vec{I}^{n} \rightarrow \vec{I}^{n}$ restricts to a map $\varphi: X \rightarrow X$ such that $\varphi\left(Y_{j_{1}, \ldots, j_{l}}\right) \subseteq X_{j_{1}, \ldots, j_{l}}$ and hence $\varphi\left(Y_{J_{1}, \ldots, J_{l}}\right) \subseteq X_{J_{1}, \ldots, J_{l}}$.

The linear homotopy $\Phi$ that connects $\varphi$ and the identity map $\operatorname{id}_{I^{n}}$ is a d-homotopy that restricts to d-homotopies on the spaces $Y_{j_{1}, \ldots, j_{l}}$ and $Y_{J_{1}, \ldots, J_{l}}$; it induces homotopies between the identity map and the maps induced by the restrictions of $\varphi$ on associated trace spaces. 
Corollary 2.13 Let $\mathbf{c}, \mathbf{d} \in X$.

(1) Every subspace $Y_{j_{1}, \ldots, j_{l}} \subset X$ is open. The path spaces $\vec{T}\left(Y_{j_{1}, \ldots, j_{l}}\right)$ (c, d) form an open cover of $\vec{T}(X)(\mathbf{c}, \mathbf{d})$.

(2) $\vec{T}\left(Y_{J_{1}, \ldots, J_{l}}\right)(\mathbf{c}, \mathbf{d})$ is contractible, resp. empty, if and only if $\vec{T}\left(X_{J_{1}, \ldots, J_{l}}\right)(\mathbf{c}, \mathbf{d})$ is contractible, resp. empty.

Proof Immediate from Proposition 2.8 and Proposition 2.12.

\section{3 (Prod)simplicial models for trace spaces}

In this and the next Section 4, we concentrate on an investigation of trace spaces $\vec{T}(X)(\mathbf{0}, \mathbf{1})$ from the bottom vertex $\mathbf{0}$ to the top vertex $\mathbf{1}$ of $X \subseteq \vec{I}^{n}$ under the further simplifying restriction that all forbidden hyperrectangles $R^{i} \subset F$ are contained in the interior of $I^{n}$. The necessary modifications arising for more general state spaces and for trace spaces of type $\vec{T}(X)(\mathbf{c}, \mathbf{d})$, resp. $\vec{T}(X)\left(\mathbf{c}, \partial_{+}(\downarrow \mathbf{d})\right)$ are discussed in Raussen [29]; these results will be published elsewhere.

\subsection{The index category $\mathcal{C}(X)(0,1)$}

3.1.1 A matrix representation of a power poset The index multisets $\left(J_{1}, \ldots, J_{l}\right)$ with $J_{i} \subseteq[1: n]$ considered in the previous Section 2 may be viewed as elements of $(\mathcal{P}([1: n]))^{l} \cong \mathcal{P}([1: l] \times[1: n])$. Elements of the latter power set can be encoded by their characteristic functions which can be viewed as binary $l \times n$-matrices:

Let $M_{l, n}=M_{l, n}(\mathbf{Z} / 2)$ denote the set of all binary $l \times n$-matrices - with $2^{l n}$ elements. Componentwise logical aut and logical and define addition and multiplication of a ring structure on $M_{l, n}$ - that does not concern us here. The total order on $\mathbf{Z} / 2$ given by $a \leq b$ unless $(a=1$ and $b=0)$ extends to a componentwise given partial order $\leq$ on $M_{l, n}$. With this partial order defining the morphisms, $M_{l, n}$ will be viewed as a poset category.

There is a natural order-preserving bijection between the subsets of $[1: l] \times[1: n]$ (elements of the power set $\mathcal{P}([1: l] \times[1: n])$ with partial order given by inclusion) and elements in $M_{l, n}$ given by

$$
J=\left(J_{1}, \ldots, J_{l}\right) \mapsto M^{J}=\left(m_{i j}^{J}\right), \quad m_{i j}^{J}=1 \Leftrightarrow j \in J_{i}
$$

with inverse $M=\left(M_{i j}\right) \mapsto J^{M}, j \in J_{i}^{M} \Leftrightarrow m_{i j}=1$.

Under this bijection, the relevant multisets $J=\left(J_{1}, \ldots, J_{l}\right)$ with $J_{i} \neq \varnothing, 1 \leq i \leq l$, correspond to matrices in the subset $M_{l, n}^{R} \subset M_{l, n}$ consisting of the $\left(2^{n}-1\right)^{l}$ matrices 
such that no row vector is a zero vector. We view $M_{l, n}^{R}$ as the full subposet category within $M_{l, n}$.

3.1.2 Subcategories and pasting functors To ease notation, we will in the following write $\vec{T}\left(X_{M}\right)(\mathbf{0}, \mathbf{1})$ instead of $\vec{T}\left(X_{J^{M}}\right)(\mathbf{0}, \mathbf{1})$. The relevant index category to consider here is the full subposet category $\mathcal{C}(X)(\mathbf{0}, \mathbf{1}) \subset M_{l, n}^{R} \subset M_{l, n}$ consisting of all matrices $M$ such that

$$
\vec{T}\left(X_{M}\right)(\mathbf{0}, \mathbf{1}) \text { is nonempty. }
$$

This index category $\mathcal{C}(X)(\mathbf{0}, \mathbf{1})$ gives rise to functors $\mathcal{D}$ and $\mathcal{E}$ into Top:

- The functor $\mathcal{D}: \mathcal{C}(X)(\mathbf{0}, \mathbf{1})^{\mathrm{op}} \rightarrow$ Top associates $\vec{T}\left(X_{M}\right)(\mathbf{0}, \mathbf{1})$ to the matrix $M$; the reverse partial order on $\mathcal{C}(X)(\mathbf{0}, \mathbf{1})$ corresponds to inclusion in Top.

- The functor $\mathcal{E}: \mathcal{C}(X)(\mathbf{0}, \mathbf{1}) \rightarrow$ Top restricts from a functor $\mathcal{E}_{n}^{l}: M_{l, n}^{R} \rightarrow$ Top; it associates to $M^{J}$ with $J=\left(J_{1}, \ldots, J_{l}\right)$ - all $J_{i} \neq \varnothing$ ! - the standard simplex product $\Delta^{\left|J_{1}\right|-1} \times \cdots \times \Delta^{\left|J_{l}\right|-1} \subset \mathbf{R}^{\left|J_{1}\right|} \times \cdots \times \mathbf{R}^{\left|J_{l}\right|} \subset\left(\mathbf{R}^{n}\right)^{l} ; \mathbf{R}^{\left|J_{i}\right|}$ is included in $\mathbf{R}^{n}$ as the subspace given by the equations $x_{j}=0, j \notin J_{i}$. For this functor, the original partial order on $\mathcal{C}(X)(\mathbf{0}, \mathbf{1})$ corresponds to inclusion in Top.

The functor $\mathcal{E}_{n}^{l}$ should be considered as a pasting scheme for the product of simplices $\left(\Delta^{n-1}\right)^{l}$; the functor $\mathcal{E}$ becomes then a pasting scheme for a subprodsimplicial complex (cf Kozlov [20]) $X_{M} \subseteq\left(\Delta^{n-1}\right)^{l}$ to be explained below.

Remark 3.1 We prefer $\mathcal{C}(X)(\mathbf{0}, \mathbf{1})$ as indexing category to the nerve of the covering given by the spaces $X_{j_{1}, \ldots, j_{l}}$, since an intersection $X_{M}$ - in view of Lemma $2.5-$ can arise in many ways as intersection of the basic spaces $X_{j_{1}, \ldots, j_{l}}$ corresponding to matrices in which every row is a unit vector; even as intersection of a varying number of the basic covering sets. The nerve of that latter covering carries redundant information: it does not take care of the product structure that gives rise to automatically commuting morphisms. It is in fact a barycentric subdivision of $\mathcal{C}(X)(\mathbf{0}, \mathbf{1})$ as will be explained below.

\subsection{Trace spaces and prodsimplicial complexes as colimits}

Regarding the functors $\mathcal{E}$ and $\mathcal{D}$ as pasting schemes, we consider their colimits:

- $\operatorname{colim}(\mathcal{D})=\vec{T}(X)(\mathbf{0}, \mathbf{1})$ by Lemma 2.10 .

- $\operatorname{colim}\left(\mathcal{E}_{n}^{l}\right)=\left(\Delta^{n-1}\right)^{l}$. 
- $\mathbf{T}(X)(\mathbf{0}, \mathbf{1}):=\operatorname{colim}(\mathcal{E}) \subset \operatorname{colim}\left(\mathcal{E}_{n}^{l}\right)=\left(\Delta^{n-1}\right)^{l}$ is a prodsimplicial complex (in the terminology of Kozlov [20]) consisting of all those products of simplices $\Delta^{\left|J_{1}\right|-1} \times \cdots \times \Delta^{\left|J_{l}\right|-1}$ that correspond to tuples $\left(J_{1}, \ldots, J_{l}\right)$ such that $M^{J} \in$ $\mathcal{C}(X)(\mathbf{0}, \mathbf{1})$; in other words, the functor $\mathcal{E}$ is a pasting scheme for a prodsimplicial complex with one simplex product for each $M \in M_{l, n}^{R}$ giving rise to a nonempty trace space $\vec{T}\left(X_{M}\right)(\mathbf{0}, \mathbf{1})$.

Remark 3.2 This prodsimplicial complex is not a general complex of morphisms in the sense of Kozlov [20, 9.2.4]. Whether $\vec{T}\left(X_{J_{1}, \ldots J_{l}}\right)(\mathbf{0}, \mathbf{1})$ is nonempty cannot be decided by investigating whether all $\vec{T}\left(X_{j_{1}, \ldots j_{l}}\right)(\mathbf{0}, \mathbf{1}), j_{i} \in J_{i}$, are nonempty. The topology of the complex does not only depend on its 1-skeleton [20, Proposition 18.1].

Comparing with $\operatorname{colim}\left(\mathcal{E}_{n}^{l}\right)=\left(\Delta^{n-1}\right)^{l}$, we obtain at once:

Lemma 3.3 The prodsimplicial complex $\mathbf{T}(X)(\mathbf{0 , 1})$ is a subcomplex of $\left(\partial \Delta^{n-1}\right)^{l} \cong$ $\left(S^{n-2}\right)^{l}$. It has at most $n^{l}$ vertices, and $\operatorname{dim}(\mathbf{T}(X)(\mathbf{0}, \mathbf{1})) \leq(n-2) l$.

Proof From Remark 2.9, it follows that $\vec{T}\left(X_{M}\right)(\mathbf{0}, \mathbf{1})=\varnothing$ as soon as $M$ has a row vector consisting of digits one only; in particular, no product can have a (full) factor $\Delta^{n-1}$. The complex $\left(\partial \Delta^{n-1}\right)^{l}$ has the number of vertices and the dimension given in the lemma.

Example 3.4 Assume that the obstruction hyperrectangles $\left.R^{i}=\right] \mathbf{a}^{i}, \mathbf{b}^{i}$ [ have the property $\mathbf{b}^{i}<\mathbf{a}^{i+1}, 1 \leq i<l$; ie, the holes are totally ordered with respect to the partial order in $\mathbf{R}^{n}$. This is the case in the first row of Example 2.3. It is not difficult to see that then $\vec{T}\left(X_{M}\right)(\mathbf{0}, \mathbf{1})=\varnothing$ if and only if $M$ has a row in which all coefficients are equal to 1 . We conclude that $\mathbf{T}(X)(\mathbf{0}, \mathbf{1})=\left(\partial \Delta^{n-1}\right)^{l} \cong\left(S^{n-2}\right)^{l}$ in this case; compare Example 4.11(4). Hence the bounds given in Lemma 3.3 are sharp!

\subsection{Homotopy equivalences}

Theorem 3.5 Assume that, for every $1 \leq i \leq n$, no upper boundary coordinate $b_{j}^{i}$ is equal to a lower boundary coordinate $a_{j}^{k}$. Then trace space $\vec{T}(X)(\mathbf{0}, \mathbf{1})$ is homotopy equivalent to the prodsimplicial complex $\mathbf{T}(X)(\mathbf{0}, \mathbf{1}) \subset\left(\partial \Delta^{n-1}\right)^{l}$ and to the nerve of the category $\mathcal{C}(X)(\mathbf{0}, \mathbf{1})$; the latter simplicial complex arises as a barycentric subdivision of $\mathbf{T}(X)(\mathbf{0}, \mathbf{1})$.

Proof First, we determine the homotopy colimits of the functors defining the pasting schemes above. We apply the homotopy lemma [20, Theorem 15.12] to the natural 
transformation $\Psi: \mathcal{D} \Rightarrow \mathcal{T}^{*}$ from $\mathcal{D}$ to the trivial functor $\mathcal{T}^{*}: \mathcal{C}(X)(\mathbf{0}, \mathbf{1})^{\text {op }} \rightarrow$ Top which sends every object into the same one-point space. Since the maps corresponding to $\Psi$ are homotopy equivalences at any object $M$ in $\mathcal{C}(X)(\mathbf{0}, \mathbf{1})$ (from a contractible space $\vec{T}\left(X_{M}\right)(\mathbf{0}, \mathbf{1})$ - by Proposition $2.8(2)$ - to a point), the map hocolim $\mathcal{D} \rightarrow$ hocolim $\mathcal{T}^{*}$ induced by $\Psi$ is a homotopy equivalence by the homotopy lemma. By definition, hocolim $\mathcal{T}^{*}$ is the nerve $\Delta(\mathcal{C}(X)(\mathbf{0}, \mathbf{1}))$ of the indexing category.

A similar argument shows that also the trivial natural transformation from $\mathcal{E}$ to $\mathcal{T}: \mathcal{C}(X)(\mathbf{0}, \mathbf{1}) \rightarrow$ Top induces a homotopy equivalence of homotopy colimits.

Next, we wish to apply the projection lemma [20, Theorem 15.19] - with two twists - to the fiber projection maps hocolim $\mathcal{D} \rightarrow \operatorname{colim} \mathcal{D}$ and hocolim $\mathcal{E} \rightarrow \operatorname{colim} \mathcal{E}$. If applicable, that lemma ensures that these projection maps are homotopy equivalences. Altogether, the maps discussed above fit to yield a homotopy equivalence

$$
\begin{aligned}
& \vec{T}(X)(\mathbf{0}, \mathbf{1})=\operatorname{colim}(\mathcal{D}) \longleftarrow \operatorname{hocolim}(\mathcal{D}) \longrightarrow \operatorname{hocolim}\left(\mathcal{T}^{*}\right) \\
& \mathbf{T}(X)(\mathbf{0}, \mathbf{1})=\operatorname{colim}(\mathcal{E}) \longleftarrow \operatorname{hocolim}(\mathcal{E}) \longrightarrow \operatorname{hocolim}(\mathcal{T})
\end{aligned}
$$

since the two opposite categories $\mathcal{C}(X)(\mathbf{0}, \mathbf{1})$ and $\mathcal{C}(X)(\mathbf{0}, \mathbf{1})^{\text {op }}$ have the same classifying space $\Delta(\mathcal{C}(X)(\mathbf{0}, \mathbf{1}))$. In particular, $\mathbf{T}(X)(\mathbf{0}, \mathbf{1})$ is also homotopy equivalent to the nerve $\Delta(\mathcal{C}(X)(\mathbf{0}, \mathbf{1}))$ - which is thus a barycentric subdivision of $\mathbf{T}(X)(\mathbf{0}, \mathbf{1})$.

The first twist alluded to above consists in using, instead of the nerve diagram of the covering given by the spaces $X_{j_{1}, \ldots, j_{l}}$, the functors $\mathcal{D}$ and $\mathcal{E}$ with the smaller indexing category $\mathcal{C}(X)(\mathbf{0}, \mathbf{1})$; cf Remark 3.1. To get to the conclusion in case of the functor $\mathcal{E}$, we use moreover, that $\mathbf{T}(X)(\mathbf{c}, \mathbf{d})$ has a prodsimplicial and thus a $\mathrm{CW}-$ structure; cf Kozlov [20, Remark 15.20].

As to the functor $\mathcal{D}$, we need to verify the conditions of the projection lemma: It was shown in Raussen [28], that $\vec{T}(X)(\mathbf{0}, \mathbf{1})$ is paracompact - even under much weaker assumptions to $X$. Furthermore, Proposition 2.12 allows us to replace the cover given by the subspaces $\vec{T}\left(X_{j_{1}, \ldots, j_{l}}\right)(\mathbf{0}, \mathbf{1})$ to that given by the homotopy equivalent open subspaces $\vec{T}\left(Y_{j_{1}, \ldots, j_{l}}\right)(\mathbf{0}, \mathbf{1})$ from Definition 2.11, with the same colimit and a homotopy equivalent homotopy colimit.

Remark 3.6 A modified version of Theorem 3.5 holds without assuming that the obstruction hyperrectangles are contained in the interior of $I^{n}$; also for trace spaces of type $\vec{T}(X)(\mathbf{c}, \mathbf{d})$ and $\vec{T}(X)\left(\mathbf{c}, \partial_{+} \downarrow \mathbf{d}\right)$ described in Section 2 . The only necessary change is a different description of the corresponding index category; this has been worked out in Raussen [29]. 
When these index categories $\mathcal{C}(X)(\mathbf{c}, \mathbf{d})$ are determined (cf Section 4 and Raussen [29, Chapter 5]) one may replace the morphism sets $\vec{T}(X)(\mathbf{c}, \mathbf{d})$ in the trace category $\vec{T}(X)$ [26] by the corresponding prodsimplicial complexes $\mathbf{T}(X)(\mathbf{c}, \mathbf{d})$ and calculate their homological invariants. In particular, their homology groups in dimension 0 describe the fundamental category of the d-space $X$.

\section{Determination of the index category}

To determine, using Theorem 3.5, the prodsimplicial model $\mathbf{T}(X)(\mathbf{0}, \mathbf{1})$ of trace space $\vec{T}(X)(\mathbf{0}, \mathbf{1})$, we need to describe the indexing category $\mathcal{C}(X)(\mathbf{0}, \mathbf{1})$ explicitly. We have to determine which of the subspaces $\vec{T}\left(X_{M}\right)(\mathbf{0}, \mathbf{1})$ corresponding to matrices $M \in M_{l, n}^{R}$ are empty and which not; cf (3-2).

It turns out that (non)emptyness can be investigated by a method that was originally designed in Fajstrup, Goubault and Raussen [5] for the detection of deadlocks and associated unsafe regions in models for the simple Higher Dimensional Automata described in Section 2.1.

A deadlock in $X$ is an element $\mathbf{x} \in X$ that admits only the constant path as d-path with source $\mathbf{x}$. The unsafe region corresponding to the deadlock $\mathbf{x}$ consists of all $\mathbf{y} \in X$ such that $n o$ d-path in $X$ with source $\mathbf{y}$ can leave the hyperrectangle spanned by $\mathbf{y}$ and $\mathbf{x}$.

It will be shown that $\vec{T}\left(X_{M}\right)(\mathbf{0}, \mathbf{1})=\varnothing$ is equivalent to the existence of a deadlock $(\neq 1)$ within $X_{M}$. This in turn depends on whether a certain set of inequalities determined by $M$ - between coordinates of the obstruction hyperrectangles $R^{i}$ holds.

A simple-minded version of the procedure worked out below was described earlier in Raussen [24]; it was restricted entirely to dimension $n=2$.

\subsection{Empty path spaces and deadlocks}

Remember the notation convention: $1 \leq i \leq l$ enumerates the obstruction hyperrectangles $R^{i} ; 1 \leq j \leq n$ enumerates the $n$ coordinate directions in $\mathbf{R}^{n}$.

We begin with a "dual" look at the spaces $X_{J_{1}, \ldots, J_{l}}$ from Definition 2.1, resp. $X_{M}$ from (3-1) in Section 3.1. For each of the original forbidden hyperrectangles $R^{i}=\prod_{j=1}^{n} I_{j}^{i}$ (cf Section 2), we define $n$ extended hyperrectangles

$$
R_{j}^{i}=\prod_{k=1}^{j-1} \widetilde{I}_{k}^{i} \times I_{j}^{i} \times \prod_{k=j+1}^{n} \widetilde{I}_{k}^{i}, \quad 1 \leq i \leq l, 1 \leq j \leq n,
$$


with $\widetilde{I}_{k}^{i}=\left[0, a_{k}^{i}\right] \cup I_{k}^{i}=\left[0, b_{k}^{i}[\right.$; an interval with 0 as its lower boundary. For illustrations, consult Figure 2 - the black rectangles are extended in several ways by white rectangles - and Figure 4 showing an extension of the forbidden black cube to a box containing also the grey-shaded part.

Remark 4.1 Each of the hyperrectangles $R_{j}^{i}$ has a lowest vertex for which all apart from one of the coordinates are 0 .

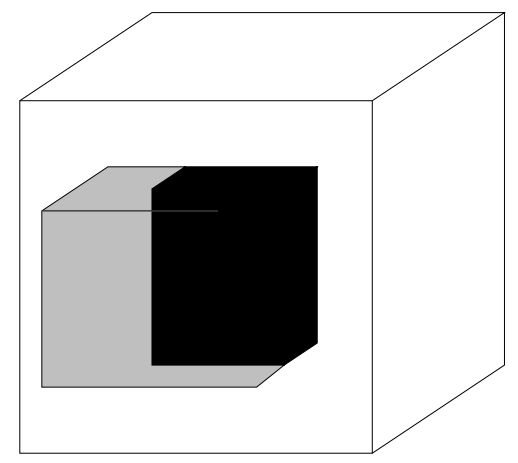

Figure 4: Extension of a three-dimensional hyperrectangle

By negating (2-2) from Definition 2.1, one obtains immediately for every matrix $M=\left(m_{i j}\right) \in M_{l, n}$ :

Lemma 4.2 $X_{M}=\vec{I}^{n} \backslash \bigcup_{m_{i j}=1} R_{j}^{i}$.

The following result shows that (non)-emptyness of the relevant trace spaces can be established by checking a bunch of inequalities. These inequalities arise via the detection of deadlocks in the subspaces $X_{M}$ by identifying nonempty intersections of $n$ extended hyperrectangles among the $R_{j}^{i}, m_{i j}=1$, and their associated unsafe regions; cf Fajstrup, Goubault and Raussen [5, Theorem 2.1].

Proposition 4.3 For a matrix $M \in M_{l, n}^{R}$, the following are equivalent:

(1) $M$ is not an object in $\mathcal{C}(X)(\mathbf{0}, \mathbf{1})$.

(2) $\vec{T}\left(X_{M}\right)(\mathbf{0}, \mathbf{1})=\varnothing$.

(3) There is a map $i:[1: n] \rightarrow[1: l]$ such that

$$
m_{i(j), j}=1 \text { for all } 1 \leq j \leq n \text { and } \bigcap_{1 \leq j \leq n} R_{j}^{i(j)} \neq \varnothing .
$$


(4) There is a map $i:[1: n] \rightarrow[1: l]$ with

$$
a_{j}^{i(j)}<b_{j}^{i(k)} \text { for all } j, k \in[1: n] .
$$

Proof The equivalence of (1) and (2) follows from the definition of $\mathcal{C}(X)(\mathbf{0}, \mathbf{1})$; cf (3-2). To establish the equivalence of (3) and (4), note that an intersection of (homothetic) hyperrectangles is nonempty if and only if each coordinate of the bottom vertex of one of the participating hyperrectangles is smaller than the corresponding coordinates of the top vertices of all the other participating hyperrectangles. From Remark 4.1 we know that all but one of the lower coordinates of the $R_{j}^{i(j)}$ are zero; the requirement has thus only to be checked for $a_{j}^{i(j)}$; exactly what is required in (4).

Assuming (2), ie $\vec{T}\left(X_{M}\right)(\mathbf{0}, \mathbf{1})=\varnothing$, the bottom vertex $\mathbf{0}$ must be contained in the unsafe region associated to a deadlock $(\neq \mathbf{1})$ for some configuration of $n$ forbidden hyperrectangles chosen among the $R_{j}^{i}, j \in J_{i}$. (If a deadlock making $\mathbf{0}$ unsafe arises by a configuration containing one or several of the original hyperrectangles $R^{i}$, then extending $R^{i}$ to some $R_{j}^{i}, m_{i j}=1$, will enlarge the compound obstruction and certainly again give rise to a configuration with the same property. Hence, we may restrict attention to configurations consisting of extended hyperrectangles only. It is important that the matrix $M \in M_{l, n}^{R}$ has no zero row vector for this argument to hold; cf also Remark 4.4 below.) The existence of a deadlock in $X_{M}$ is equivalent to the existence of a nonempty intersection $\bigcap_{1 \leq j \leq n} R_{j}^{i(j)}$ (see Fajstrup, Goubault and Raussen [5, Theorem 2.1]), ie of a map as given in (3).

On the other hand, granted (3), if $\bigcap_{1 \leq j \leq n} R_{j}^{i(j)} \neq \varnothing$, the intersection gives rise to a deadlock $\mathbf{e}=\left(e_{1}, \ldots, e_{n}\right) \neq \mathbf{1}$ in $X_{M}$; in fact the coordinates $e_{j}$ of a are maximal among the $j$-th coordinates of the $R_{j}^{i(j)}$; in our case $e_{j}=a_{j}^{i(j)}$, cf [5, Theorem 2.1]. The associated unsafe region has as its bottom vertex the point in $X$ the $n$ coordinates of which are next to maximal among these lower coordinates of the $R_{j}^{i(j)}, 1 \leq j \leq n$ [5, Theorem 2.2].

Now we exploit that the extended hyperrectangles are special (cf Remark 4.1), in the sense that all these coordinates (next to maximal among the lower coordinates) are 0 ! Hence $\mathbf{0}$ is automatically in the unsafe region associated to (any) deadlock $\mathbf{e} \neq \mathbf{1}$ in $X_{M}$. In particular, there is no d-path with source $\mathbf{0}$ leaving $\downarrow$ e. This proves (2): $\vec{T}\left(X_{M}\right)(\mathbf{0}, \mathbf{1})=\varnothing$.

Remark 4.4 In proving (2) implies (3) above, it is crucial that all index sets $J_{i}$ are nonempty. Otherwise, a number of extended hyperrectangles $R_{j}^{i}$ might, together with some of the original $R^{i}$, generate a deadlock with $\mathbf{0}$ in the unsafe region that does not arise from a nonempty intersection of extended hyperrectangles. Figure 5 gives an illustration for that phenomenon in dimension two. 


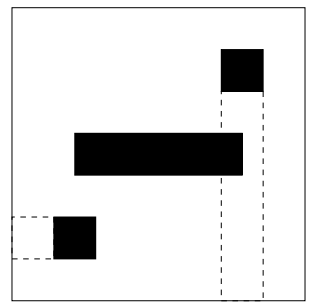

Figure 5: Deadlock arising from a combination of extended and nonextended rectangles

\subsection{Algorithmic determination of $\mathcal{C}(X)(0,1)$}

4.2.1 The map $\Psi$ and its properties We will also consider the following subset of the set of binary matrices in $M_{l, n}$ :

- $M_{l, n}^{C} \subset M_{l, n}$ consists of the matrices such that every column vector is a unit vector - they form a subset with $l^{n}$ elements. Every such matrix $M$ represents the characteristic function of the graph of some map $i:[1: n] \rightarrow[1: l]$; cf Proposition 4.3(3-4).

To $i:[1: n] \rightarrow[1: l]$, we associate the matrix $M(i) \in M_{l, n}^{C}$ whose only nonzero coefficients are given as $m_{i(j), j}$.

Define the map $\Psi: M_{l, n} \rightarrow \mathbf{Z} / 2$ by $\Psi(M)=1 \Leftrightarrow \vec{T}\left(X_{M}\right)(\mathbf{0}, \mathbf{1})=\varnothing$; equivalently, $\Psi(M)=0 \Leftrightarrow M \in \mathcal{C}(X)(\mathbf{0}, \mathbf{1})$ for matrices $M \in M_{l, n}^{R}$.

Proposition $4.5 \quad$ (1) $\Psi$ is order-preserving.

(2) $\Psi(M)=0$ if $M \in M_{l, n}$ has a zero vector among its column vectors.

(3) $\Psi(M)=1 \Leftrightarrow$ there exists $N \in M_{l, n}^{C}$ with $\Psi(N)=1$ and $N \leq M \in M_{l, n}$.

Proof (1) If $M \leq M^{\prime} \in M_{l, n}$, then $\vec{T}\left(X_{M^{\prime}}\right)(\mathbf{0}, \mathbf{1}) \subseteq \vec{T}\left(X_{M}\right)(\mathbf{0}, \mathbf{1})$. If the latter set is empty, the first set needs to be empty, as well.

(2) Assume that the $j$-th column in $M$ is the zero vector. Then no obstruction hyperrectangle $R^{i}$ is extended in direction $j$. Hence, all $j$-th lower coordinates chosen from the extended hyperrectangles corresponding to $M$ are strictly positive. In particular, $\mathbf{0}$ is not contained in the unsafe region of any deadlock occurring in $X_{M}$; in particular, there exists a d-path from $\mathbf{0}$ to 1 since neither the lower face $x_{j}=0$ in $I^{n}$ nor the upper boundary $\partial_{+} \downarrow \mathbf{1}$ intersect any of the extended hyperrectangles.

(3) One implication is an immediate consequence of (1). 
For the other implication, we may assume in view of (2) that a matrix $M$ with $\Psi(M)=1$ has no zero vector among its column vectors. By Proposition 4.3(3), there is a map $i:[1: n] \rightarrow[1: l]$ giving rise to a deadlock with $\mathbf{0}$ in the associated unsafe region. In particular, we conclude for the associated matrix $N=M(i) \in M_{l, n}^{C}$ with $M(i) \leq M: \vec{T}\left(X_{M(i)}\right)(\mathbf{0}, \mathbf{1})=\varnothing$ and hence $\Psi(M(i))=1$.

The determination of $\Psi$ can thus be performed in two steps. First, we determine the restriction of $\Psi$ to the subset $M_{l, n}^{C}$ consisting of matrices $M(i)$ that correspond to maps $i:[1: n] \rightarrow[1: l]$. In particular, we determine the set of matrices ( $D$ for "dead")

$$
D(X)(\mathbf{0}, \mathbf{1}):=\left\{M \in M_{l, n}^{C} \mid \Psi(M)=1\right\} .
$$

Using this set $D(X)(\mathbf{0}, \mathbf{1})$, we will then apply Proposition 4.5(3) to determine the set of matrices

$$
\mathcal{C}(X)(\mathbf{0}, \mathbf{1}):=\left\{M \in M_{l, n}^{R} \mid \Psi(M)=0\right\} .
$$

describing the objects of the relevant index category.

4.2.2 Determination of $D(X)(\mathbf{0}, \mathbf{1})$ To a matrix $M \in M_{l, n}^{C}$, we associate its row set $R(M):=\left\{1 \leq i \leq l \mid \mathbf{m}^{i} \neq \mathbf{0}\right\} \subseteq[1: l]$ - indexing the nonzero rows $\mathbf{m}^{i}$ of $M$. The row set $R(M(i))$ is equal to the image $i([1: n]) \subseteq[1: l]$.

The condition from Proposition 4.3(4) leads us to consider the same upper bounds $b_{j}^{i(k)}$ for matrices $M$ with the same row set $R(M)=B \subseteq[1: l]$ : To each of the

$$
\sum_{k=1}^{\min (n, l)}\left(\begin{array}{l}
n \\
k
\end{array}\right) \leq 2^{l}-1
$$

nonempty subsets $B \subseteq[1: l]$ of cardinality at $\operatorname{most} \min (n, l)$ corresponds an upper bound $\mathbf{b}^{B}=\left(b_{1}^{r_{1}}, \ldots, b_{n}^{r_{n}}\right) \in[0,1]^{n}$ with $b_{j}^{r_{j}}=\min _{i \in B} b_{j}^{i}$.

Ordering the $j$-th coordinates $a_{j}^{i}$, resp. $b_{j}^{i}$ of subinterval boundaries for $\vec{I}_{j}^{i} \subset \vec{I}_{j}$ (eg by a quicksort algorithm) gives rise to $2 n$ (not necessarily well-determined) permutations $\pi_{j}^{0}, \pi_{j}^{1} \in \Sigma_{l}$ such that

$$
a_{j}^{\pi_{j}^{0}(1)} \leq \cdots \leq a_{j}^{\pi_{j}^{0}(n)} \quad \text { and } \quad b_{j}^{\pi_{j}^{1}(1)} \leq \cdots \leq b_{j}^{\pi_{j}^{1}(n)} .
$$

A comparison of these two ordered lists leads to maps $C_{j}:[1: l] \rightarrow[1: l]$ given by

$$
C_{j}(k):=\max \left\{r \mid 1 \leq r \leq l, a_{j}^{\pi_{j}^{0}(r)}<b_{j}^{\pi_{j}^{1}(k)}\right\}, \quad 1 \leq j \leq n .
$$

Note that $C_{j}(k) \geq k$ for all $j$ and that $C_{j}$ is monotone. Only the relative order of the $a_{j}^{i}, b_{j}^{i}$ matters! 
Every $n$-tuple $\left(r_{1}, \ldots, r_{n}\right)$ in $[1: l]^{n}$ corresponds to a "mixed vertex"

$$
\left(a_{1}^{\pi_{1}^{0}\left(r_{1}\right)}, \ldots, a_{n}^{\pi_{n}^{0}\left(r_{n}\right)}\right)
$$

likewise $\left(s_{1}, \ldots s_{n}\right)$ corresponds to

$$
\left(b_{1}^{\pi_{1}^{1}\left(s_{1}\right)}, \ldots, b_{n}^{\pi_{n}^{1}\left(s_{n}\right)}\right) .
$$

The upper bound $\mathbf{b}^{B}$ corresponding to a nonempty (row) subset $B \subseteq[1: l]$ is determined by the (componentwise) minimum $\left(s_{1}^{B}, \ldots, s_{n}^{B}\right)$ of the $\left(s_{1}^{i}, \ldots, s_{n}^{i}\right)$ corresponding to the $\mathbf{b}^{i}, i \in B$; these upper bounds can be calculated recursively.

For such a nonempty row set $B \subseteq[1: l]$, let

$$
\widetilde{R}_{j}(B):=\left\{i \in[1: l] \mid a_{j}^{i}<b_{j}^{\pi^{1}\left(s_{j}^{B}\right)}\right\}=\pi_{j}^{0}\left(\left[1: C_{j}\left(s_{j}^{B}\right)\right]\right)
$$

and $R_{j}(B):=\widetilde{R}_{j}(B) \cap B$. From condition (4) in Proposition 4.3, we conclude:

Lemma 4.6 A map $i:[1: n] \rightarrow[1: l]$ gives rise to a matrix $M=M(i) \in D(X)(\mathbf{0}, \mathbf{1})$ if and only if

$$
i(j) \in R_{j}(i([1: n])) \text { for every } 1 \leq j \leq n .
$$

What is left is to describe a method that determines the sets $R_{j}(B)$ for every nonempty subset $B \subseteq[1: l]$ of cardinality at most $n$. For the determination of the sets $R_{j}(B)$, the following properties - in particular (4) - are helpful:

Lemma 4.7 (1) If $B=\{i\}$ is a one-element set, then $R_{j}(B)=B$ for $j \in[1: n]$. Hence $\Psi(M)=1$ for each of the $l$ matrices $M \in M_{l, n}^{C}$ with a one-element row set $R(M)$.

(2) $\varnothing \neq B \subseteq C \subseteq[1: l] \Rightarrow \widetilde{R}_{j}(B) \supseteq \widetilde{R}_{j}(C), 1 \leq j \leq n$.

(3) $R_{j}(B \cup C)=\left(R_{j}(B) \cap \widetilde{R}_{j}(C)\right) \cup\left(\widetilde{R}_{j}(B) \cap R_{j}(C)\right)$.

(4) For $i \notin B$, let $\mathbf{b}^{B}$ correspond to $\left(s_{1}^{B}, \ldots, s_{n}^{B}\right), \mathbf{b}^{i}$ to $\left(s_{1}^{i}, \ldots s_{n}^{i}\right)$ and $\mathbf{a}^{i}$ to $\left(r_{1}^{i}, \ldots r_{n}^{i}\right)$. Then

$$
R_{j}(B \cup\{i\})= \begin{cases}R_{j}(B), & C_{j}\left(s_{j}^{B}\right)<r_{j}^{i}, \\ R_{j}(B) \cup\{i\}, & r_{j}^{i}<C_{j}\left(s_{j}^{B}\right), s_{j}^{B}<s_{j}^{i}, \\ \left(\pi_{j}^{0}\left(\left[1: C_{j}\left(s_{j}^{i}\right)\right]\right) \cap R_{j}(B)\right) \cup\{i\}, & s_{j}^{i}<s_{j}^{B} .\end{cases}
$$

Proof (1) follows from (the proof of) Lemma 3.3; (2) is obvious. For (3), note that $R_{j}(B \cup C)=\left(\widetilde{R}_{j}(B) \cap \widetilde{R}_{j}(C)\right) \cap(B \cup C)$ and use distributivity. (4) is an easy consequence. 
4.2.3 Determination of $\mathcal{C}(X)(\mathbf{0}, \mathbf{1})$ From Proposition 4.5 and Lemma 4.6, we can conclude immediately:

\section{Proposition 4.8 Let $M \in M_{l, n}^{R}$.}

(1) $\Psi(M)=1$ if and only if there is a matrix $N \in D(X)(\mathbf{0}, \mathbf{1})$ (cf Lemma 4.6) such that $n_{i j} \leq m_{i j}$ for all $1 \leq i \leq l, 1 \leq j \leq n$.

(2) $\Psi(M)=0 \Leftrightarrow M \in \mathcal{C}(X)(\mathbf{0}, \mathbf{1})$ if and only if, for every matrix $N \in D(X)(\mathbf{0}, \mathbf{1})$, there is a pair $(i, j) \in[1: l] \times[1: n]$ such that $m_{i j}=0, n_{i j}=1$.

Matrices that are maximal with respect to the partial order $\leq$ on binary matrices within $\mathcal{C}(X)(\mathbf{0}, \mathbf{1})$ constitute - by definition - the subset $\mathcal{C}_{\max }(X)(\mathbf{0}, \mathbf{1}) \subseteq \mathcal{C}(X)(\mathbf{0}, \mathbf{1})$. By Proposition $4.5(1)$ they determine the index category $\mathcal{C}(X)(\mathbf{0}, \mathbf{1})$. They correspond to maximal simplex products in the prodsimplicial space $\mathbf{T}(X)(\mathbf{0}, \mathbf{1})$.

To determine the matrices contained in these two sets, we consider (choice) subsets $C \subseteq[1: l] \times[1: n]$ characterized by the property:

- For every matrix $N \in D(X)(\mathbf{0}, \mathbf{1})$ there exists $(i, j) \in C$ with $n_{i j}=1$.

Remark that one index $(i, j)$ can count for several matrices $N$.

Functions $m_{C}=1-\chi(C)$ for such choices are then exactly the characteristic functions for matrices $M_{C}=\left(m_{i j}\right) \in \mathcal{C}(X)(\mathbf{0}, \mathbf{1})$.

A choice $C$ is minimal, if for every $C^{\prime} \subset C$ there is a matrix $N \in D(X)(\mathbf{0}, \mathbf{1})$ with $n_{i j}=0$ for each $(i, j) \in C^{\prime}$. The function $m_{C}=1-\chi(C)$ for a minimal choice function is then the characteristic function of a maximal matrix $M_{C} \in \mathcal{C}_{\max }(X)(\mathbf{0}, \mathbf{1})$.

We describe a simple-minded algorithm constructing $\mathcal{C}_{\max }(X)(\mathbf{0}, \mathbf{1})$ step by step given $D(X)(\mathbf{0}, \mathbf{1})=\left\{D_{1}, \ldots, D_{p}\right\}$ starting with $A_{\max }^{0}(X)(\mathbf{0}, \mathbf{1})$ with the matrix $\mathbf{1}$ consisting of only $1 \mathrm{~s}$ as the only element. Assume $A_{\max }^{h-1}(X)(\mathbf{0}, \mathbf{1})=\left\{M_{1}, \ldots, M_{q_{h-1}}\right\}$ to consist of the maximal binary matrices $M$ such that $N_{k} \not \leq M$ for $1 \leq k \leq h-1<p$.

Compare the matrices $M_{l} \in A_{\max }^{h-1}(X)(\mathbf{0}, \mathbf{1})$ to $N_{h}$. If $N_{h} \not \leq M_{l}$, then keep $M_{l}$ unchanged as an element of $A_{\max }^{h}(X)(\mathbf{0}, \mathbf{1})$; if $M(i)=N_{h} \leq M_{l}$ (cf Section 4.2.1), then replace $M_{l}$ by the $n$ matrices $M_{l}^{1}, \ldots M_{l}^{n} \in A_{\max }^{h}(X)(\mathbf{0}, \mathbf{1})$ determined as follows: $M_{l}^{j}$ arises from $M_{l}$ by replacing $m_{i(j), j}^{l}=1$ in $M_{l}$ by $m_{i(j), j}^{l, j}=0$ in $M_{l}^{j}$.

Assessing whether $N \leq M$ is easy, given $N$ : form the binary product $\bigwedge_{n_{p q}=1} m_{p q}$; this product is always over $n$ entries for $N \in M_{l, n}^{C}$.

The maximal matrices in $\mathcal{C}_{\max }(X)(\mathbf{0}, \mathbf{1})$ correspond to the maximal simplex products that are patched together in $\mathbf{T}(X)(\mathbf{0}, \mathbf{1}) \subset\left(\Delta^{n-1}\right)^{l}$ while the matrices in $D(X)(\mathbf{0}, \mathbf{1})$ 
correspond to minimal nonfaces in $\left(\Delta^{n-1}\right)^{l}$. The construction above reminds of a similar construction of a simplicial complex $K(\mathcal{F})$ associated to a set system $\mathcal{F}$ used for topological investigations of colouring problems; cf eg Matoušek and Ziegler [21]; in our case, we have the product structure in the underlying category $M_{l, n}$ as an additional feature.

Corollary 4.9 Let $C \subset[1: l] \times[1: n]$ denote a choice subset.

(1) The simplex product in $\mathbf{T}(X)(\mathbf{0}, \mathbf{1})$ corresponding to $M_{c} \in \mathcal{C}(X)(\mathbf{0}, \mathbf{1})$ considered as abject in $\mathcal{C}(X)(\mathbf{0}, \mathbf{1})$ (cf Section 3.2) has dimension $(n-1) l-|C|$.

(2) $\operatorname{dim} \mathbf{T}(X)(\mathbf{0}, \mathbf{1})=(n-1) l-\min |C|$.

Proof The simplex product corresponding to $M_{C}$ has type $\prod_{1 \leq i \leq l} \Delta^{n-1-c_{i}}$ with $c_{i}=|\{j \mid(i, j) \in C\}|$.

Corollary 4.10 The Lusternik-Schnirelmann category of trace space $\vec{T}(X)(\mathbf{0}, \mathbf{1})$ satisfies the inequality cat $(\vec{T}(X)(\mathbf{0}, \mathbf{1})) \leq\left|\mathcal{C}_{\max }(X)(\mathbf{0}, \mathbf{1})\right|$.

Proof The prodsimplicial complex $\mathbf{T}(X)(\mathbf{0}, \mathbf{1})$ homotopy-equivalent to $\vec{T}(X)(\mathbf{0}, \mathbf{1})$ is covered by maximal products of simplices; there are $\left|\mathcal{C}_{\max }(X)(\mathbf{0}, \mathbf{1})\right|$ of those. As products of simplices, they are contractible; they are deformation retracts of contractible open neighbourhoods in $\mathbf{T}(X)(\mathbf{0}, \mathbf{1})$.

\subsubsection{Examples}

Example 4.11 (1) $X$ is a square with two square holes as in the first row of Figure 2, Example 2.3: $D(X)(\mathbf{0}, \mathbf{1})$ consists then of the two matrices

$$
\left[\begin{array}{ll}
1 & 1 \\
0 & 0
\end{array}\right] \text { and }\left[\begin{array}{ll}
0 & 0 \\
1 & 1
\end{array}\right]
$$

since a deadlock arises only if a rectangle is extended in both directions from the same obstruction. There are then four minimal choices $C \subset[1: 2] \times[1: 2]$; each of them has two elements. Hence $\mathcal{C}_{\max }(X)(\mathbf{0}, \mathbf{1})=\mathcal{C}(X)(\mathbf{0}, \mathbf{1})$ consists of the four matrices

$$
\left[\begin{array}{ll}
0 & 1 \\
0 & 1
\end{array}\right], \quad\left[\begin{array}{ll}
0 & 1 \\
1 & 0
\end{array}\right], \quad\left[\begin{array}{ll}
1 & 0 \\
0 & 1
\end{array}\right], \quad\left[\begin{array}{ll}
1 & 0 \\
1 & 0
\end{array}\right] \text {. }
$$

Extending each of the rectangles in one direction according to the recipe encoded in one of these matrices yields the subspaces shown in the upper row of Figure 2. Each of them allows exactly one d-homotopy class around the (extended) holes. 
The corresponding prodsimplicial complex $\mathbf{T}(X)(\mathbf{0}, \mathbf{1})$ of type $\left(\partial \Delta^{1}\right)^{2}$ consists of four points.

(2) $X$ is a square with two square holes as in the second row of Figure 2, Example 2.3: This time, $D(X)(\mathbf{0}, \mathbf{1})$ consists of the three matrices

$$
\left[\begin{array}{ll}
1 & 1 \\
0 & 0
\end{array}\right], \quad\left[\begin{array}{ll}
0 & 0 \\
1 & 1
\end{array}\right], \quad\left[\begin{array}{ll}
0 & 1 \\
1 & 0
\end{array}\right]:
$$

one additional deadlock configuration corresponding to $X_{12}$ comes up with one extension for every hole. This time, there are only three minimal choices $C$, each of cardinality 2 , corresponding to the matrices

$$
\left[\begin{array}{ll}
0 & 1 \\
0 & 1
\end{array}\right], \quad\left[\begin{array}{ll}
1 & 0 \\
0 & 1
\end{array}\right], \quad\left[\begin{array}{ll}
1 & 0 \\
1 & 0
\end{array}\right]
$$

in $\mathcal{C}_{\max }(X)(\mathbf{0}, \mathbf{1})=\mathcal{C}(X)(\mathbf{0}, \mathbf{1}) . \mathbf{T}(X)(\mathbf{0}, \mathbf{1})$ consists thus of three points.

(3) $X$ a square with three holes as in Figure 5: In this case,

$$
D(X)(\mathbf{0}, \mathbf{1})=\left\{\left[\begin{array}{ll}
1 & 1 \\
0 & 0 \\
0 & 0
\end{array}\right],\left[\begin{array}{ll}
0 & 1 \\
1 & 0 \\
0 & 0
\end{array}\right],\left[\begin{array}{ll}
0 & 0 \\
1 & 1 \\
0 & 0
\end{array}\right],\left[\begin{array}{ll}
0 & 0 \\
0 & 1 \\
1 & 0
\end{array}\right],\left[\begin{array}{ll}
0 & 0 \\
0 & 0 \\
1 & 1
\end{array}\right]\right\} .
$$

There are four minimal choices giving rise to the matrices in

$$
\mathcal{C}_{\max }(X)(\mathbf{0}, \mathbf{1})=\left\{\left[\begin{array}{ll}
1 & 0 \\
1 & 0 \\
1 & 0
\end{array}\right],\left[\begin{array}{ll}
1 & 0 \\
1 & 0 \\
0 & 1
\end{array}\right],\left[\begin{array}{ll}
1 & 0 \\
0 & 1 \\
0 & 1
\end{array}\right],\left[\begin{array}{ll}
0 & 1 \\
0 & 1 \\
0 & 1
\end{array}\right]\right\} .
$$

The complex $\mathbf{T}(X)(\mathbf{0}, \mathbf{1})$ consists of four points.

(4) $X=\vec{I}^{n} \backslash \vec{J}^{n}$ as in Example 2.4: In this case, $D(X)(\mathbf{0}, \mathbf{1})$ has only one element, the 1 -row matrix $[1,1, \ldots, 1]$. The $n$ minimal choices correspond to the one row matrices with exactly one entry 0 in $\mathcal{C}_{\max }(X)(\mathbf{0}, \mathbf{1})$. These matrices correspond to the maximal simplices in $\mathbf{T}(X)(\mathbf{0}, \mathbf{1})=\partial \Delta^{n-1}$. Products of spheres arise likewise in the case considered in Example 3.4.

We conclude from Theorem 3.5:

Corollary 4.12 $\vec{T}\left(\vec{I}^{n} \backslash \vec{J}^{n}\right)(\mathbf{0}, \mathbf{1}) \simeq \partial \Delta^{n-1}$.

Previous attempts to prove Corollary 4.12 directly were far more complicated. 
Example 4.13 (1) For the state space $X$ from Figure 1, $D(X)=M_{2,3}^{C}$ : Since the two forbidden boxes intersect, every set of extensions in all three directions will produce a deadlock and hence an empty trace space. As a consequence,

$$
\mathcal{C}_{\max }(X)(\mathbf{0}, \mathbf{1})=\left\{\left[\begin{array}{lll}
0 & 1 & 1 \\
0 & 1 & 1
\end{array}\right],\left[\begin{array}{lll}
1 & 0 & 1 \\
1 & 0 & 1
\end{array}\right],\left[\begin{array}{lll}
1 & 1 & 0 \\
1 & 1 & 0
\end{array}\right]\right\}
$$

Hence, $\mathbf{T}(X)(\mathbf{0}, \mathbf{1})$ is the union of the three diagonal squares in a flat 2-dimensional torus $\left(\partial \Delta^{2}\right)^{2}$ covered by three times three squares. In particular, $\mathbf{T}(X)(\mathbf{0}, \mathbf{1})$ is homotopy equivalent to a circle $S^{1}$.

This example can be generalized as follows: Let $X=I^{n} \backslash F$ with the forbidden region $F=\bigcup_{i=1}^{l} R^{i}$ consisting of $l \leq n$ hyperrectangles $R^{i}$ with nonempty intersection $\bigcap_{i=1}^{l} R^{i}$. Then, $\mathbf{T}(X)(\mathbf{0}, \mathbf{1})=\bigcup_{f}\left(\partial \Delta^{n-1} \backslash f\right)^{l}$ with $f$ ranging over the $n$ faces of $\partial \Delta^{n-1}$. That latter trace space is homotopy equivalent to $S^{n-2}$ - and the homotopy type is thus independent of the number $l$ of contributing hyperrectangles - by the following argument: Consider the diagonal inclusion

$$
i: S^{n-2} \simeq \partial \Delta^{n-1}=\bigcup_{f} \partial \Delta^{n-1} \backslash f \hookrightarrow \bigcup_{f}\left(\partial \Delta^{n-1} \backslash f\right)^{l}=\mathbf{T}(X)(\mathbf{0}, \mathbf{1}) .
$$

Both spaces are colimits of contractible spaces over the same poset index category given by the nonempty proper subsets of $[1: n]$ (subsets of faces), and the inclusion respects this filtration. The result follows thus from the homotopy lemma [20, Theorem 15.12].

More generally, it seems promising to use the intersection pattern among the contributing hyperrectangles as an input simplifying the determination of the index categories.

(2) The space $X$ in Figure 6 below shows a cube from which two wedges, each of them composed of two intersecting rectangular boxes are removed. Remark that the two wedges do not touch each other. The trace in that drawing from bottom to top is homotopic but not dihomotopic (homotopic through a 1-parameter deformation of d-paths) to a trace on the boundary of the cube. A simple-minded analysis of this model in Raussen [25] showed by a quite intricate argument that the trace space for this d-space (from bottom to top) is not connected.

The general method described in this article yields a model for trace space $\vec{T}(X)(\mathbf{0}, \mathbf{1})$ as a subspace of the 4-torus $\left(\partial \Delta^{2}\right)^{4} \cong\left(S^{1}\right)^{4}$. It turns out by inspection (similar to (1) above) that one can handle each of the two wedges as one obstruction in this case. It turns out that the trace space can then be seen as the union of five squares and a disjoint extra ("corner") point in the two-torus $\left(\partial \Delta^{2}\right)^{2}=\left(S^{1}\right)^{2}$ on the right hand side of Figure 6 above. This subspace is of course homotopy equivalent to the disjoint union of a wedge of circles and of an extra point: $\vec{T}(X)(\mathbf{0}, \mathbf{1}) \simeq\left(S^{1} \vee S^{1}\right) \sqcup *$. 

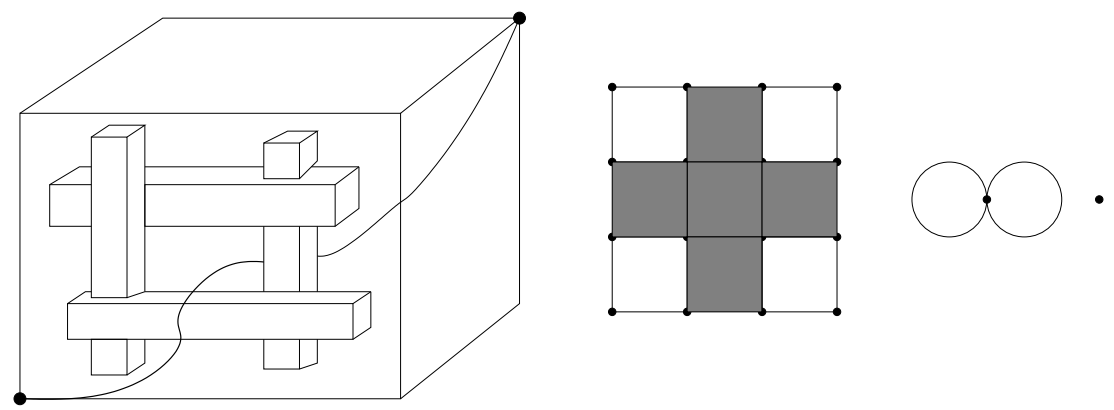

Figure 6: State space $X$ and associated models for trace space $\vec{T}(X)(\mathbf{0}, \mathbf{1})$

It would be interesting to find more general methods for dimension reduction as the one described above.

4.2.5 A reformulation: Minimal transversals in hypergraphs The search for minimal choices in $D(X)(\mathbf{0}, \mathbf{1})$ can be translated into a well-known and well-investigated problem in combinatorics. ${ }^{1}$ The set $D(X)(\mathbf{0}, \mathbf{1})$ may be considered as a hypergraph (with hyperedges $=$ simplices connecting a number of vertices; every matrix in $D(X)(\mathbf{0}, \mathbf{1})$ defines a hyperedge $)$ on the vertex set $[1: l] \times[1: n]$. A minimal choice - that allows to find the maximal elements in $\mathcal{C}_{\max }(\mathbf{0}, \mathbf{1})$; cf Section 4.2 .3 - is then a minimal transversal (or hitting set) of that hypergraph: This means that it has nonempty intersection with every hyperedge and it is minimal with this property. Computing minimal transversals has many applications (eg, machine learning, indexing of databases, data mining and optimization). There are several articles about algorithms for finding minimal transversals and their complexity in the literature; cf eg Khachiyan, Boros, Elbassioni and Gurvich [19].

The hypergraph given by the matrices in $D(X)(\mathbf{0}, \mathbf{1})$ has special properties: All hyperedges have the same cardinality $n$; even more so, they are graphs of functions from $[1: n]$ to $[1: l]$. This ought to simplify the setting.

\subsection{Homology of the trace space}

By Theorem 3.5, the homology of the trace space $\vec{T}(X)(\mathbf{0}, \mathbf{1})$ may be calculated as the homology of the associated prodsimplicial complex $\mathbf{T}(X)(\mathbf{0}, \mathbf{1})$. Given the poset category $\mathcal{C}(X)(\mathbf{0}, \mathbf{1})$, this is the homology of a particular chain complex $C(X)(\mathbf{0}, \mathbf{1})$ with one generator for every product of simplices.

\footnotetext{
${ }^{1}$ I would like to thank my colleague Leif Kjær Jørgensen, Aalborg University, for mentioning hypergraphs and their transversals to me.
} 
More precisely, let $C_{k}(X)(\mathbf{0}, \mathbf{1})$ denote the free $R$-module generated by all matrices in $\mathcal{C}(X)(\mathbf{0}, \mathbf{1})$ with $(k+l)$ entries $1 ; R$ denotes the chosen coefficient ring. For a matrix $M \in M_{l, n}$ with $m_{p q}=1$, let $M_{p q}$ be given by

$$
\left(m_{p q}\right)_{i j}= \begin{cases}m_{i j} & (i, j) \neq(p, q), \\ 0 & (i, j)=(p, q) .\end{cases}
$$

The boundary operator $\partial$ on $C(X)(\mathbf{0}, \mathbf{1})$ is then given by

$$
\partial(M)=\sum_{m_{p q}=1}(-1)^{|(p, q)|} M_{p q}
$$

with alternating sign: the integer $|(p, q)|=\sum_{i=1}^{p-1} \sum_{j=1}^{n} m_{i j}+\sum_{j=1}^{q} m_{p j}-1$ takes account of the ones in $M$ preceding $m_{p q}=1$.

It should be interesting to perform actual homology calculations in "real life" examples that give rise to huge chain complexes. The algorithms for the calculation of homology in Kaczynski, Mrozek and Slusarek [18] by reduction of chain complexes (with field coefficients) might be helpful. Likewise a modification of the algorithms in Kaczynski, Mischaikow and Mrozek [17] for the homology of cubical complexes.

\section{Models for more general trace spaces}

\subsection{Trace spaces corresponding to concurrent nonlinear programs}

So far, we have only looked at model spaces corresponding to concurrent linear programs; without branchings, mergings and loops. More realistic models can be investigated using the same tools - but with a twist: Let $\Gamma=\prod_{j=1}^{n} \Gamma_{j}$ denote a product of directed graphs (branchings, mergings and loops allowed); each $\Gamma_{j}$ represents a program run by a single processor. The graphs $\Gamma_{j}$ are regarded as d-spaces (realizations of precubical sets of dimension one), and $\Gamma$ is given the product structure: as an $n-$ dimensional precubical complex with d-space structure (cf Grandis [12]).

A directed interval $J_{j}$ from $a_{j}$ to $b_{j}$ in the geometric realization of a component $\Gamma_{j}$ is uniquely given by the image $p_{j}(I)$ of a trace $p_{j} \in \vec{T}\left(\Gamma_{j}\right)\left(a_{j}, b_{j}\right)$ arising from an injective d-path; no loops allowed here! Remark on the other hand, that such a directed interval is not always determined uniquely by its end points.

A (generalized) hyperrectangle in $\Gamma$ is a product $R=\prod_{j} J_{j} \subseteq \prod_{j} \Gamma_{j}=\Gamma$ of such directed intervals. A forbidden region $F=\bigcup_{i} R^{i}$ is a union of such generalized hyperrectangles, and the state space $X=\Gamma \backslash F$ is its complement. 
The aim is to analyse the space of d-paths $\vec{P}(X)(\mathbf{x}, \mathbf{y}) \subseteq \vec{P}(\Gamma)(\mathbf{x}, \mathbf{y})$ (or the space of traces $\vec{T}(X)(\mathbf{x}, \mathbf{y})$ homotopy equivalent to it) between two points $\mathbf{x}=\left(x_{1}, x_{2}, \ldots, x_{n}\right)$ and $\mathbf{y}=\left(y_{1}, y_{2}, \ldots, y_{n}\right)$ in the space $X$. First, we have a look at the (bigger) space $\vec{T}(\Gamma)(\mathbf{x}, \mathbf{y})$ and then, we will use the map induced on traces by the inclusion map $i_{X}: X \hookrightarrow \Gamma$.

For a directed graph - no cubes of higher dimension supporting homotopies are available - dihomotopy of d-paths (with fixed end points) is equivalent to reparametrization equivalence; cf Fahrenberg and Raussen [4]. In particular, each factor $\vec{T}\left(\Gamma_{j}\right)\left(x_{j}, y_{j}\right)$ is discrete; every component is represented by a (reparametrization equivalence class of) a particular directed path from $x_{j}$ to $y_{j}$. The product structure of $\Gamma$ yields:

Lemma 5.1 $\vec{T}(\Gamma)(\mathbf{x}, \mathbf{y}) \simeq \prod \vec{T}\left(\Gamma_{j}\right)\left(x_{j}, y_{j}\right)$. In particular, $\vec{T}(\Gamma)(\mathbf{x}, \mathbf{y})$ is homotopy discrete.

Proof The d-space structure and the dihomotopy relations factor:

$$
\vec{T}(\Gamma) \simeq \vec{P}(\Gamma) \cong \prod \vec{P}\left(\Gamma_{j}\right) \simeq \prod \vec{T}\left(\Gamma_{j}\right) .
$$

Remark 5.2 To enumerate the components (=traces) of the space of d-paths in a directed graph $\Gamma$, one should first reduce $\Gamma$ to normal form $N(\Gamma)$ : Vertices with valency $(1,-1)$ - exactly one ingoing and one outgoing arrow, different from each other - are suppressed; the two arrows are concatenated to one. The normal form $N(\Gamma)$ does no longer have such vertices.

Attach a unique label to each arrow in a directed graph $\Gamma$ in normal form and form words in these labels along concatenable arrows. Then $\vec{T}(\Gamma)$ corresponds to the discrete set of such words; $\vec{T}(\Gamma)(x, y)$ to the words starting and ending with one or several specific labels, depending on whether $x, y$ correspond to vertices or to points on a directed edge. There is no need to distinguish between points on the interior of the same edge.

Each component $C \in \vec{T}(\Gamma)(\mathbf{x}, \mathbf{y})$ can thus be represented by an $n$-tuple of (traces of) specific d-paths $c_{j} \in \vec{P}\left(\Gamma_{j}\right)\left(x_{j}, y_{j}\right)$. As representatives, we choose $c_{j} \in \vec{R}\left(\Gamma_{j}\right)\left(x_{j}, y_{j}\right) \subset$ $\vec{P}\left(\Gamma_{j}\right)\left(x_{j}, y_{j}\right)$ to be regular (ie, locally injective; cf Fahrenberg and Raussen [4])); every other d-path in $\vec{P}\left(\Gamma_{j}\right)\left(x_{j}, y_{j}\right)$ dihomotopic to $c_{j}$ is then a reparametrization $c_{j} \circ \varphi_{j}$ of $c_{j}$ with $\varphi_{j} \in \vec{P}(\vec{I})(0,1)$ an (increasing) d-path in the standard ordered unit interval $\vec{I}$ [4, Theorem 3.6 and Proposition 3.8]. The d-paths $c_{j}$ altogether define a dmap (a specific "delooping") $c: \vec{I}^{n} \rightarrow \Gamma$ given as $c\left(t_{1}, \ldots, t_{n}\right)=\left(c_{1}\left(t_{1}\right), \ldots, c_{n}\left(t_{n}\right)\right)$, and: 
Lemma 5.3 The $d$-map $c: \vec{I}^{n} \rightarrow \Gamma$ induces a homeomorphism

$$
c \circ: \vec{T}\left(\vec{I}^{n}\right)(\mathbf{0}, \mathbf{1}) \rightarrow C \subset \vec{T}(\Gamma)(\mathbf{x}, \mathbf{y}), p \mapsto c \circ p .
$$

Comparing d-paths in $\Gamma$ and in $X=\Gamma \backslash F$ and given such a component $C \in \pi_{0}(\vec{T}(\Gamma)) \cong$ $\prod \pi_{0}\left(\vec{T}\left(\Gamma_{j}\right)\right)$, the following two questions arise naturally:

(1) Does $C$ lift to $X$ at all (ie, can it be represented by an - interleaving - d-path in $X$ rather than in $\Gamma)$ ?

(2) Determine the topology of $i_{X}^{-1}(C)$, ie, of the space of all d-paths in $X$ whose projections to the $\Gamma_{j}$ are (reparametrizations of) these specified execution paths ("interleavings").

Every directed interval $J=] a_{j}, b_{j}\left[\subset \Gamma_{j}\right.$ (in the sense above) pulls back to the standard interval $c_{j}^{-1}(] a_{j}, b_{j}[) \subset I-$ which is an open subinterval of $I$ in the subspace topology, possibly empty. To each generalized hyperrectangle $R^{i}=\prod J_{j}^{i} \subset \Gamma$ corresponds thus an (honest) hyperrectangle $\widetilde{R}^{i}=c^{-1}\left(R^{i}\right)=\prod c_{j}^{-1}\left(J_{j}^{i}\right)$, possibly empty. The forbidden region $F \subset \Gamma$ corresponds to a forbidden region $\widetilde{F}=c^{-1}(F)=\bigcup_{i} \widetilde{R}^{i} \subset I^{n}$, leaving $\tilde{X}=I^{n} \backslash \tilde{F} \subset I^{n}$ as state space (with the d-structure inherited from $\vec{I}^{n}$ ). By restricting the homeomorphism $c \circ$ from Lemma 5.3, we obtain

Corollary 5.4 The d-map $c: \tilde{X} \rightarrow X$ induces a homeomorphism

$$
c \circ: \vec{T}(\tilde{X})(\mathbf{0}, \mathbf{1}) \rightarrow i_{X}^{-1}(C) \subset \vec{T}(X)(\mathbf{x}, \mathbf{y}) .
$$

Example 5.5 Let $\Gamma_{1}=\vec{S}^{1}$ denote a circular digraph and $\Gamma_{2}=\vec{I}$ a linear one. Let $X=\left(\Gamma_{1} \times \Gamma_{2}\right) \backslash J^{2}$ with $J^{2} \subset\left(\Gamma_{1} \times \Gamma_{2}\right)$ an open rectangular hole; cf Figure 7 . The component $C_{r}$ in $\vec{T}\left(\Gamma_{1} \times \Gamma_{2}\right)(\mathbf{0}, \mathbf{1})$ corresponding to $r+1 / 2$ spiral tours leads to a state space $\tilde{X}_{r}$ with $r$ rectangular holes with an exponential covering map back to $X$ :
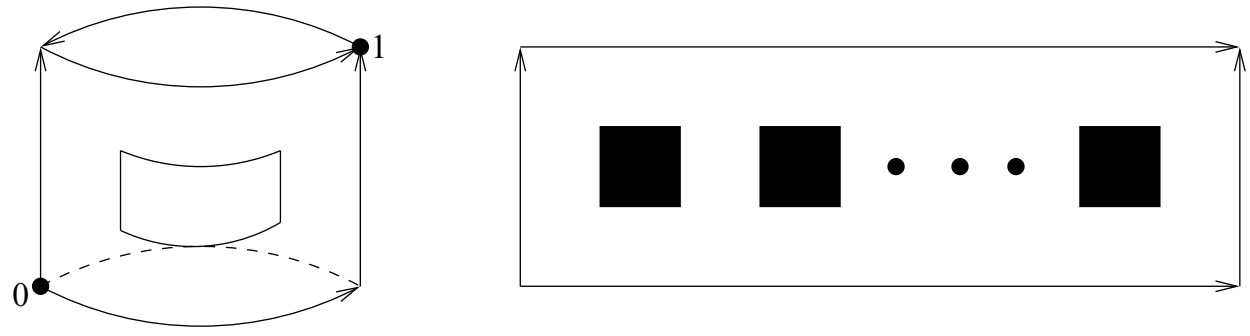

Figure 7: State spaces $X$ (directed cylinder with hole) and $\tilde{X}_{r}$ 
The contractible(!) components of $\vec{T}(X)(\mathbf{0}, \mathbf{1})$ in this case correspond to pairs $(r, s)$ of integers $r \geq s \geq 0$. The integer $r$ counts the number of rounds (the delooping); the integer $s$ describes where a d-path in the component passes from the lower to the upper annulus.

Corollary 5.4 allows us to attack the questions asked above:

(1) This question is equivalent to: Is $\vec{T}(\tilde{X})(\mathbf{0}, \mathbf{1})$ nonempty? This is the case if $\mathbf{0}$ is not contained in the unsafe region corresponding to any deadlock in $\tilde{X}-$ this can be settled using the techniques described in Fajstrup, Goubault and Raussen [5]; compare also Fajstrup and Sokolowski [8].

(2) The topology of a nonempty space $i_{X}^{-1}(C) \cong \vec{T}(\tilde{X})(\mathbf{0}, \mathbf{1})$ can be analysed as that of the prodsimplicial complex $\mathbf{T}(\tilde{X})(\mathbf{0}, \mathbf{1})$ as described in Section 4.

Remark 5.6 The components $C \subset \vec{T}(\Gamma)(-,-)$ form the morphisms of the fundamental category $\vec{\pi}_{1}(\Gamma)$ (composition induced by concatenation of paths) with the elements of $\Gamma$ as objects. In particular, loop components act on (the left and on the right) on components with matching end points. In [8], Fajstrup and Sokolowski have shown that unsafe areas corresponding to a specific deadlock point can look quite different for components ("deloopings") with the same end points. It should be interesting to investigate how the topology of the spaces $i_{X}^{-1}(C)$ behaves under composition with loops. For applications, it is essential to find out whether there is an algorithm determining them in a recursive fashion.

\subsection{Simplicial models for trace spaces in precubical complexes}

The methods used in this paper can certainly be applied more generally. General Higher Dimensional Automata can be described as labelled precubical complexes with a compatible d-structure defined originally for every single cube; cf Fajstrup, Goubault and Raussen [5]. In Raussen [28], we investigated spaces of d-paths in a non-selflinked precubical complex $X$ and showed that, for all $x_{0}, x_{1} \in X$, the path spaces $\vec{P}(X)\left(x_{0}, x_{1}\right)$ are ELCX (equi locally convex) in the sense of Milnor [22]; hence that they are locally contractible and possess the homotopy type of a CW-complex.

The main ingredient in the proof is the construction of a locally defined average map $\mu: U \rightarrow X$ defined on a neighbourhood $U=\bigcup_{\beta} V_{\beta} \times V_{\beta}$ of the diagonal with $V_{\beta}$ the open star neighbourhood of a vertex $\beta$ in $X$. This average map plays a role very similar to that of the least upper bound $\vee$ in Section 2.

In particular, a directed sequence of adjacent vertices and their open star neighbourhoods gives rise to a contractible space of d-paths (or traces) progressing consecutively through 
that sequence of neighbourhoods. The spaces of d-paths in all possible such sequences give rise to a covering of the space of all d-paths (with given end points) by contractible subspaces [28, Proposition 3.16]. Using the same method (and restrictions of the map $\mu$ above), it can be shown that intersections of such subspaces (through intersections of open stars of certain vertices) are empty or also contractible.

The nerve lemma [20, Theorem 15.21] shows then that spaces of d-paths (and thus of traces) in a precubical complex (with given end points) are homotopy equivalent to the nerve of the covering described above. In particular, $\vec{T}(X)\left(x_{0}, x_{1}\right)$ has an explicit structure of a simplicial complex. To describe it explicitly, one needs to know which sets of sequences of adjacent vertices give rise to open star neighbourhoods with a (common) d-path contained in each of them.

Remark 5.7 More abstractly, one may describe a category of contractible cube paths in semicubical complexes (with contractible trace spaces and such that all subcube paths are contractible, as well) and then consider the induced category over $X$ (objects = semicubical maps from a contractible cube path into $X$ respecting given end points). The nerve of that category (or of any subcategory that covers all d-paths in $X$ with given end points) is then homotopy equivalent to $\vec{T}(X)\left(x_{0}, x_{1}\right)$.

\section{References}

[1] R Brown, P J Higgins, Colimit theorems for relative homotopy groups, J. Pure Appl. Algebra 22 (1981) 11-41 MR621285

[2] R Brown, P J Higgins, On the algebra of cubes, J. Pure Appl. Algebra 21 (1981) 233-260 MR617135

[3] E W Dijkstra, Co-operating sequential processes, from: "Programming Languages", (F Genuys, editor), Academic Press, New York (1968) 43-110

[4] U Fahrenberg, M Raussen, Reparametrizations of continuous paths, J. Homotopy Relat. Struct. 2 (2007) 93-117 MR2369163

[5] L Fajstrup, E Goubault, M Raussen, Detecting deadlocks in concurrent systems, from: "CONCUR'98: concurrency theory (Nice)", (D Sangiorgi, R de Simone, editors), Lecture Notes in Comput. Sci. 1466, Springer, Berlin (1998) 332-347 MR1683333

[6] L Fajstrup, M Raussen, E Goubault, Algebraic topology and concurrency, Theoret. Comput. Sci. 357 (2006) 241-278 MR2242768

[7] L Fajstrup, M Raussen, E Goubault, E Haucourt, Components of the fundamental category. Homotopy theory, Appl. Categ. Structures 12 (2004) 81-108 MR2057412

[8] L Fajstrup, S Sokolowski, Infinitely running concurrents processes with loops from a geometric viewpoint, Elec. Notes Theor. Comput. Sci. 39 (2000) 
[9] RJ van Glabbeek, On the expressiveness of higher dimensional automata, Theoret. Comput. Sci. 356 (2006) 265-290 MR2223695

[10] E Goubault, E Haucourt, Components of the fundamental category. II, Appl. Categ. Structures 15 (2007) 387-414 MR2350213

[11] M Grandis, Directed homotopy theory. II. Homotopy constructs, Theory Appl. Categ. 10 (2002) No. 14, 369-391 MR1921751

[12] M Grandis, Directed homotopy theory. I, Cah. Topol. Géom. Différ. Catég. 44 (2003) 281-316 MR2030049

[13] M Grandis, Directed algebraic topology. Models of non-reversible worlds, New Math. Monogr. 13, Cambridge Univ. Press (2009) MR2562859

[14] J Gunawardena, Homotopy and concurrency, Bull. EATCS 54 (1994) 184-193

[15] M Herlihy, S Rajsbaum, Algebraic topology and distributed computing-a primer, from: "Computer science today", (J van Leeuwen, editor), Lecture Notes in Comput. Sci. 1000, Springer, Berlin (1995) 203-217 MR1389577

[16] J F Jardine, Path categories and resolutions, Preprint (2009) Available at http:// www.math. uwo.ca/ jardine/papers/preprints/pathcat-paper3.pdf

[17] T Kaczynski, K Mischaikow, M Mrozek, Computational homology, Applied Math. Sciences 157, Springer, New York (2004) MR2028588

[18] T Kaczynski, M Mrozek, M Ślusarek, Homology computation by reduction of chain complexes, Comput. Math. Appl. 35 (1998) 59-70 MR1604791

[19] L Khachiyan, E Boros, K Elbassioni, V Gurvich, A new algorithm for the hypergraph transversal problem, from: "Computing and combinatorics", (L Wang, editor), Lecture Notes in Comput. Sci. 3595, Springer, Berlin (2005) 767-776 MR2190900

[20] D Kozlov, Combinatorial algebraic topology, Algorithms and Computation in Math. 21, Springer, Berlin (2008) MR2361455

[21] J Matoušek, G M Ziegler, Topological lower bounds for the chromatic number: a hierarchy, Jahresber. Deutsch. Math.-Verein. 106 (2004) 71-90 MR2073516

[22] J Milnor, On spaces having the homotopy type of a CW-complex, Trans. Amer. Math. Soc. 90 (1959) 272-280 MR0100267

[23] V Pratt, Modeling concurrency with geometry, from: "POPL '91: Proceedings of the 18th ACM SIGPLAN-SIGACT symposium on Principles of programming languages”, ACM, New York (1991) 311-322

[24] M Raussen, On the classification of dipaths in geometric models for concurrency. Geometry and concurrency, Math. Structures Comput. Sci. 10 (2000) 427-457 MR1786470

[25] M Raussen, Deadlocks and dihomotopy in mutual exclusion models, Theoret. Comput. Sci. 365 (2006) 247-257 MR2269456 
[26] M Raussen, Invariants of directed spaces, Appl. Categ. Structures 15 (2007) 355-386 MR2350212

[27] M Raussen, Reparametrizations with given stop data, J. Homotopy Relat. Struct. 4 (2009) 1-5 MR2481616

[28] M Raussen, Trace spaces in a pre-cubical complex, Topology Appl. 156 (2009) 17181728 MR2521708

[29] M Raussen, Simplicial models for trace spaces, Tech. Report R-2010-02, Dept. of Math. Sciences, Aalborg University (2010) Available at http://www.math.aau.dk/ fileadmin/user_upload/www.math.aau.dk/Forskning/Rappor\% tserien/ $\mathrm{R}-2010-02 \cdot \mathrm{pdf}$

[30] G Winskel, M Nielsen, Models for concurrency, from: "Handbook of logic in computer science, Vol. 4", (S Abramsky, D M Gabbay, T S E Maibaum, editors), Handb. Log. Comput. Sci. 4, Oxford Univ. Press, New York (1995) 1-148 MR1365754

Department of Mathematical Sciences, Aalborg University Fredrik Bajersvej 7G, DK-9220 Aalborg Øst, Denmark

raussen@math. aau. dk

http://people.math.aau.dk/ raussen

Received: 30 March 2010 